\title{
Genome-wide identification of the Phaseolus vulgaris sRNAome using small RNA and degradome sequencing
}

\author{
Damien Formey ${ }^{1}$, Luis Pedro Iñiguez ${ }^{1}$, Pablo Peláez², Yong-Fang Li ${ }^{3}$, Ramanjulu Sunkar ${ }^{3}$, Federico Sánchez², \\ José Luis Reyes ${ }^{2^{*}}$ and Georgina Hernández ${ }^{1 *}$
}

\begin{abstract}
Background: MiRNAs and phasiRNAs are negative regulators of gene expression. These small RNAs have been extensively studied in plant model species but only 10 mature microRNAs are present in miRBase version 21, the most used miRNA database, and no phasiRNAs have been identified for the model legume Phaseolus vulgaris. Thanks to the recent availability of the first version of the common bean genome, degradome data and small RNA libraries, we are able to present here a catalog of the microRNAs and phasiRNAs for this organism and, particularly, we suggest new protagonists in the symbiotic nodulation events.

Results: We identified a set of 185 mature miRNAs, including 121 previously unpublished sequences, encoded by 307 precursors and distributed in 98 families. Degradome data allowed us to identify a total of 181 targets for these miRNAs. We reveal two regulatory networks involving conserved miRNAs: those known to play crucial roles in the establishment of nodules, and novel miRNAs present only in common bean, suggesting a specific role for these sequences. In addition, we identified 125 loci that potentially produce phased small RNAs, with 47 of them having all the characteristics of being triggered by a total of 31 miRNAs, including 14 new miRNAs identified in this study.

Conclusions: We provide here a set of new small RNAs that contribute to the broader knowledge of the sRNAome of Phaseolus vulgaris. Thanks to the identification of the miRNA targets from degradome analysis and the construction of regulatory networks between the mature microRNAs, we present here the probable functional regulation associated with the sRNAome and, particularly, in $\mathrm{N}_{2}$-fixing symbiotic nodules.
\end{abstract}

Keywords: Phaseolus vulgaris, microRNAs, phasiRNAs, Degradome, Nodules, Legumes, Common bean

\section{Background}

Phaseolus vulgaris, known as common bean, is the most important legume for human consumption. This crop is the principal source of protein for hundreds of millions of people and more than 18 million tonnes of dry common bean are produced annually [1]. As a legume, $P$. vulgaris is also a model species for the study of symbiosis in association with nitrogen-fixing bacteria in the genus Rhizobium. The recent release of the common bean genome sequence [2] allows the research

\footnotetext{
*Correspondence: jlreyes@ibt.unam.mx; gina@ccg.unam.mx

${ }^{2}$ Departamento de Biología Molecular de Plantas, Instituto de Biotecnología (UNAM), Av. Universidad 2001, Cuernavaca 62210, Morelos, Mexico ${ }^{1}$ Centro de Ciencias Genómicas, Universidad Nacional Autónoma de México (UNAM), Av. Universidad 1001, Cuernavaca 62210, Morelos, Mexico Full list of author information is available at the end of the article
}

community to extend their analyses and acquire needed knowledge about this organism. In recent years, a number of studies have focused on gene expression in common bean and its role in a broad range of processes [3] including the response to biotic $[4,5]$ or abiotic $[6,7]$ stresses. Gene regulation has particularly been investigated at the post-transcriptional level with the action of small regulating elements called microRNAs (miRNAs) [8-11].

The regulatory processes performed by miRNAs are widely conserved in plants, animals, protists and fungi [12-15], highlighting the significant influence that these regulators can have on the evolution of gene expression. The miRNAs are small non-coding RNA sequences of $22 \mathrm{nt}$ that negatively regulate gene expression, usually, post-transcriptionally by base-pairing to complementary 
transcripts. In plants, these small RNAs are processed from longer hairpin-shaped precursors encoded in the genome and almost all of them are transcribed by RNA polymerase II. This pri-miRNA, for primary miRNA transcript, is first processed into a precursor, the premiRNA, and then excised as a RNA duplex by the endonuclease enzyme Dicer-like 1 (DCL1). The resulting duplex sequence, composed by the guide miRNA and the complementary miRNA* destined for degradation, is then exported to the cytoplasm by diverse factors, including the HASTY protein. Current knowledge is lacking about the spatio-temporal separation of the two strands but, once performed, the guide miRNA is loaded on to a member of the AGO protein family to assemble the RNA-induced silencing complex (RISC). The miRNA can then play its regulatory role by specifically binding a target transcript based on sequence complementarity. To date, three types of action have been identified for plant miRNAs: cleavage, leading to the degradation of the corresponding transcripts; translational inhibition, disrupting protein production; and in some cases DNA methylation, preventing the transcription of the corresponding genomic locus. In fine, the miRNA action leads to the loss of function of the gene by inhibiting protein production (see Voinnet [16] for review).

Plant miRNAs are involved in most, if not all, biological processes and have been found in all the organs where they have been searched for [17]. These sequences are key regulators in important processes such as hormone regulation, nutrient homeostasis, development and interaction with pathogens and symbionts [18-22]. As part of some of these mechanisms and processes, miRNAs can act indirectly on gene regulation via triggering the production of other small RNAs. These molecules are called phasiRNAs, for phased small interfering RNAs, including the tasiRNAs (trans-acting siRNAs) and other phased small RNAs that require cleavage by miRNAs [23]. Particular transcripts cleaved by microRNAs are recruited by the RNA-dependent RNA polymerase RDR6 and SGS3 to generate double-stranded RNA molecules [24] that are subsequently processed by DCL4 or DCL5 into phasiRNAs of 21 or 24 nt, respectively $[25,26]$. Similar to what occurs with miRNAs, these molecules can be loaded on to AGO proteincontaining complexes and can direct disruption of protein production by transcript targets, including transcripts distinct from their own production source, but still exhibiting sequence complementarity [27]. Most of the phasiRNAs are produced from protein-coding transcripts and, if not, they are derived from long non-coding mRNAs. Many phasiRNAs target, and are derived from, large protein families such as NB-LRR, MYB and PPR proteins. One recently hypothesized role of phasiRNAs is to target NB-LRR proteins that broadly regulate plant defenses and beneficial microbial interactions [27], which are important features for model legumes such as common bean.

To date, around 10000 miRNAs have been identified in all the Viridiplantae organisms reported in miRBase version 21 [28]. Despite several studies on this topic [29-31], only 8 precursors of miRNAs, generating 10 mature sequences, are referenced for $P$. vulgaris in this database while more than 500 are present for other model legumes such as Medicago truncatula or Glycine max. The phasiRNAs have not been studied in this organism. Here, we use the recently released genome of common bean [2], 5 small RNA libraries obtained from 5 plant organs, and degradome sequencing to identify a high confidence genome-wide common bean miRNA dataset, the associated target transcripts, and the first $P$. vulgaris phasiRNA catalog ever published.

\section{Results and discussion}

\section{Overview of the sequencing data}

In this study, we used four published high-throughput sequencing libraries of small RNAs [29] obtained from flowers, leaves, seedlings and roots of Phaseolus vulgaris and a novel library acquired from symbiotic nodules of the same organism obtained by infection with Rhizobium tropici. To identify the whole set of miRNAs and phasiRNAs, we performed this analysis with the recently described genome of Phaseolus vulgaris (Phaseolus vulgaris v1.0, DOE-JGI and USDA-NIFA, http://www.phytozome. net/commonbean) [2] as a reference. For the plant organ libraries and the symbiotic nodules, we obtained averages of $3,649,274$ and 2,810,685 reads, respectively (Table 1 ). From these sets, an average of 51 and $37 \%$ of reads were matched to the common bean genome, respectively (Table 1), and $8.3 \%$ of the sequences from the nodule library matched with the corresponding bacterial genome (Rhizobium tropici CIAT899, [32]). The lower percentage of reads mapping to the plant genome in the nodule library was partly due to the presence of bacterial sequences and to an increased abundance of ligated adaptor sequences in this particular sample.

\section{Identification and organ distribution of miRNAs}

To identify the already referenced and novel miRNAs of Phaseolus vulgaris, we used the miRDeep-P pipeline [33] with each library as a set of small RNA candidates and the $P$. vulgaris genome as the corresponding reference.

Table 1 Statistics of the 5 small RNA library genomic mapping

\begin{tabular}{llllll}
\hline & Filower & Leave & Root & Seedling & Nodule \\
\hline Raw reads & 3356817 & 3321526 & 3963174 & 3955581 & 2810685 \\
Mapped reads & 1756842 & 1663979 & 1950627 & 2138258 & 1032364 \\
\% of mapped reads & $52 \%$ & $5 \%$ & $49 \%$ & $54 \%$ & $37 \%$ \\
\hline
\end{tabular}


We identified a total of 307 precursors that fulfilled our criteria (see methods) producing 185 unique mature miRNAs (Table 2). 64 of them are already referenced as mature miRNAs in other plant species (miRBase database ver. 21 [28]): these are produced by 111 precursors and distributed throughout 27 families. In this work, we refer to those sequences as "known" miRNAs. Additionally, 57 miRNAs are new isoforms (or family members) [34] of already referenced miRNAs in the miRBase database: these are generated by 98 precursors and distributed in 25 families (Additional file 1: Table S1). We refer to them as "new isomiRs". Finally, we identified 64 novel miRNAs that are not members of previously described miRNA families. These novel miRNAs are encoded by 98 precursors, grouped in 59 families.

In summary, we identified a total of 185 mature miRNAs encoded by 307 precursors and distributed in 98 families. These microRNAs included 64 already known miRNAs, 57 novel isoforms belonging to known miRNA families and a last set of 64 novel miRNAs not identified before. Among the 40 families already registered in miRBase 21 that we have identified (Additional file 1: Table S1), we retrieved all those conserved within the angiosperm genomes, which are the miRNAs from miR156 to miR408 [35]. As expected, the highly conserved miR482, together with the more restricted miR1512 and miR1515, which have been reported as positively regulating nodule number [36], are also present in our dataset. Part of the other identified miRNA families belong to the so-called "legume" miRNAs [37] such as the miR2111, miR2118 and miR2119. Other families retrieved in our libraries had only been identified, up to now, in G. $\max$ (miR4415, miR4416 and miR5786), M. truncatula (miR2597) or both (miR5037). Thanks to the identification of these miRNAs in P. vulgaris, we observe that they are now shared between at least two legume species and can properly be called "legume" miRNAs [37]. Interestingly, we also found 5 members of the miR1862 family and 1 member of the

Table 2 Statistics of the identified precursors and their corresponding mature miRNAs

\begin{tabular}{lllll}
\hline & Known & New isomiR & Novel & Total \\
\hline Identified precursors & 111 & 98 & 98 & 307 \\
Identified miRNAs & 64 & 57 & 64 & 185 \\
Relative position & & & & \\
\hline Intergenic & 97 & 90 & 78 & 265 \\
Intron & 1 & 5 & 16 & 22 \\
Exon & 11 & 3 & 4 & 18 \\
3'UTR & 2 & 0 & 0 & 2 \\
5'UTR & 0 & 0 & 0 & 0 \\
\hline
\end{tabular}

miR6175 that so far have only been identified in rice [38] and rubber tree [39], respectively. The members of these two families are sparsely expressed in our libraries and we can imagine that these miRNAs, and others characterized by exhibiting low expression, are highly spatially- or temporally-specific sequences. It is possible that this type of "specific" miRNA is, in fact, present in several organisms and, with the increase in the depth of high-throughput sequencing technologies, will begin to emerge in investigations focused on the identification of miRNAs in less-studied organisms. The miR4376 family may be an example of these. It has been shown to be a super-family derived from the miR390 and was probably present in the common ancestor of Spermatophyta [40], suggesting that this miRNA is conserved in most of the seed plants, but currently only identified in five species: soybean, tomato, ginseng, potato and, now, common bean (miRBase 21).

By sequencing five small RNA libraries corresponding to five different plant organs, i.e., flower, leaf, nodule, root and seedling, we are able to indicate the distribution of the identified miRNAs in the whole plant based on their expression (see methods). As expected, we found about $61 \%$ of the known miRNAs (39/64) present in all of the organs while only about $14 \%$ and $12 \%$ of the new isomiRs $(8 / 57)$ and the novel miRNAs (8/64), respectively, are in this class (Fig. 1). In contrast, 22 novel miRNAs are organ-specific whereas none of the known miRNA is. This suggests that the known miRNAs, composed of a majority of conserved miRNAs, play a role at the whole-plant level, as expected from their implication in regulatory networks [41]. In contrast, the newly identified miRNAs, considered as recently emerged and, probably having a more specific action (or no action at all) are characterized by a specific spatial distribution [42]. Alternatively, the characteristic lower expression of isomiRs and novel miRNAs may preclude accurate detection in all organs tested, thus limiting this analysis.

The analysis of miRNA expression and distribution in different organs has been used to identify nodulespecific novel miRNAs in G. max and M. truncatula [43, 44]. Similar approaches used in this work led us to identify three nodule-specific miRNAs in P. vulgaris. These include the new isoform of a conserved miRNA family, the miRNov627 from the mtr-miR399 family and two newly identified miRNAs: miRNov153 and miRNov494. Their putative targets are involved in the regulation of plant-microorganism interactions, which will be discussed below. Other miRNAs with organ-specific expression patterns were detected in our samples and could be also regulating specific processes in other plant tissues (Additional file 2: Table S2). 

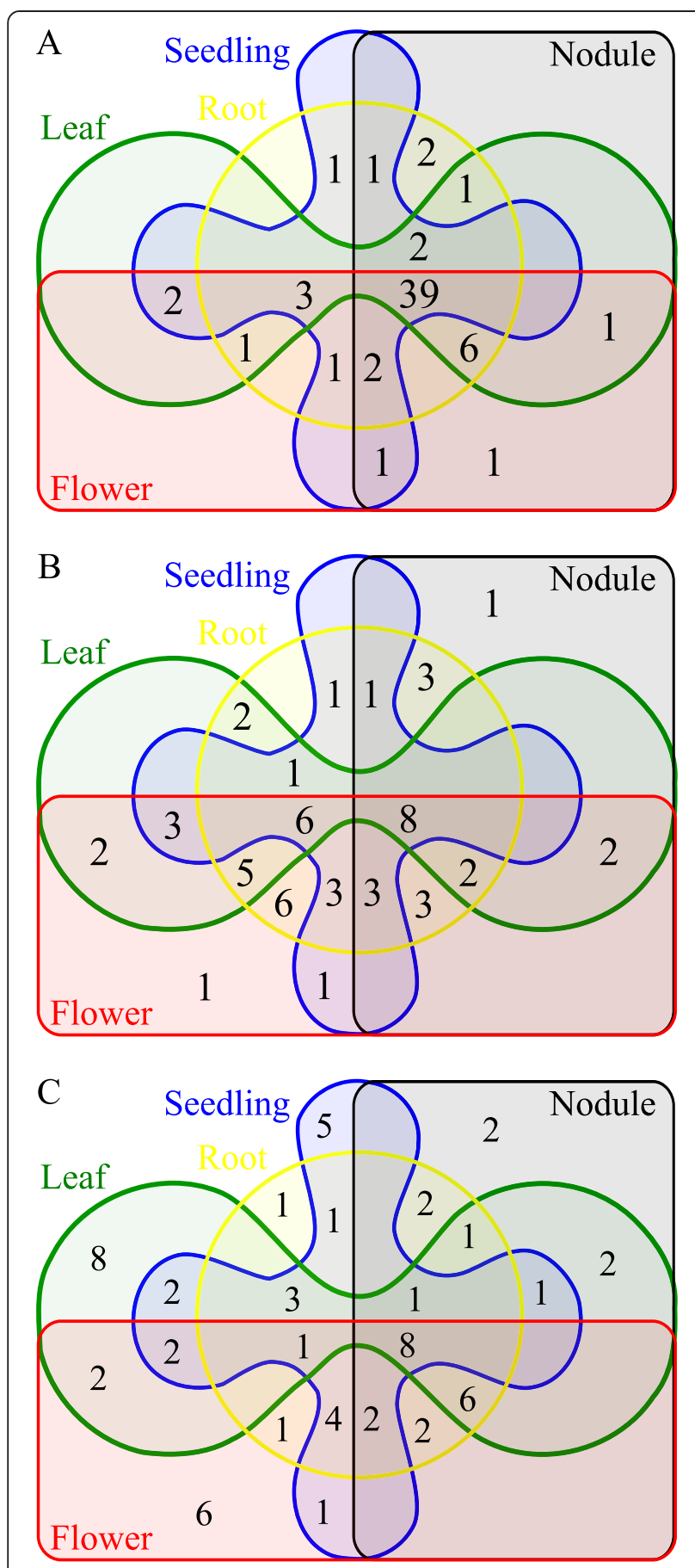

Fig. 1 Distribution of the identified mature miRNAs in 5 plant organs. Venn diagram of the distribution of $\mathbf{a}$ known miRNA, $\mathbf{b}$ new isomiR and c novel miRNA in the different studied organs. Red: flower, green: leaf, yellow: root, blue: seedling and black: nodule. The numbers in each Venn diagram area correspond to the number of mature miRNAs encountered in the corresponding overlapping organ area

\section{Validation of the predicted precursors}

The recent release of the $P$. vulgaris gene atlas [45] allowed us to verify that the predicted miRNAs have a corresponding transcript in the 24 transcriptome data samples collected from seven distinct tissues of common bean at developmentally important time-points, including plants inoculated with either effective or ineffective Rhizobium. We found that about 65,26 and $24 \%$ of the known miRNA, new isomiR and novel miRNA precursors, respectively, have a complete transcript in at least one of the 24 common bean expression libraries. These validated precursors produce 84,47 and $35 \%$ of the known, new isomiR and novel mature sequences. To differentiate miRNAs from other small RNAs, we used degradome data to identify the specific signatures of DCL slicing during the miRNA precursor processing. These signatures are characterized by the finding of a significant processing event at the mature miRNA and/ or miRNA* boundaries within the precursor [46]. With this method, we validated the correct processing of $41 \%$ of the known miRNA precursors, $30 \%$ of the new isomiR precursors and $22 \%$ of the novel miRNA precursors, thus constituting the validation of $63 \%$ of the known mature miRNAs, $44 \%$ of the new mature isomiRs and $33 \%$ of the novel mature miRNAs (Fig. 2). The failure to validate some miRNA precursors could be due to undesirable RNA degradation producing random degradome signatures, or the presence of degradome signatures originating from other loci that mask the expected significant signatures [47]. It is also possible that some precursors are not functional and mature miRNAs only originate from a subset of potential precursors within the same MIR family. Compared with the precursors encoding well-conserved miRNAs, a lower proportion of precursors for novel miRNAs allowed degradome validation. A general problem encountered with these sequences is their lower expression levels and corresponding lower degradome signatures, the latter being obscured by other gene degradation products.

Finally, if we combine the results of the expression data with those of the degradome analysis, we obtain supporting data for 78,47 and $35 \%$ of the known, new isomiRs and novel miRNA precursors we encountered, respectively, representing 92,54 and $44 \%$ of the corresponding mature miRNAs originally identified. Overall, $64 \%$ of the identified mature microRNAs show evidence of expression for at least one of their proposed precursors by at least one of the two methods examined.

\section{miRNA identification aided by genomic sequence data}

The present study profited from the use of the recently released complete genomic sequence for $P$. vulgaris [2] to identify miRNAs. Compared to the previous study of Peláez et al. [29], this new approach allowed us to confirm the presence of 81 of the 113 known miRNAs identified by Peláez et al. in P. vulgaris. 25 out of the 32 miRNAs previously identified by Peláez et al. and not encountered in our study are actually not present in the 

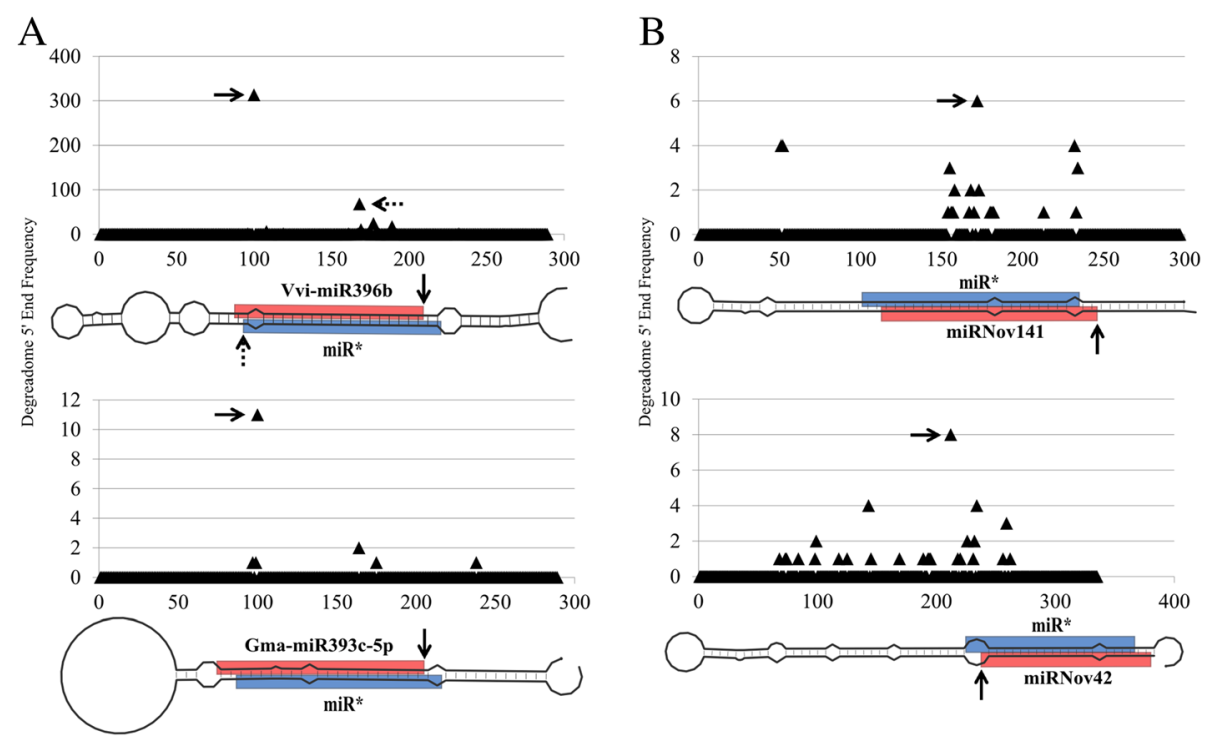

Fig. 2 Distribution of degradome signatures on miRNA precursors. Distribution of degradome read abundance on a known miRNA and $\mathbf{b}$ novel miRNA precursors. Vertical axes display the read abundance and the horizontal axes display the precursor position by base pair. The black triangles show the read abundance for the corresponding precursor base. The plain and dotted arrows point to the first base of the mature miRNA and the first base of the miR*, respectively. The schemes below the plots represent the corresponding precursors. The vertical bars show the base pairing and the red and blue boxes represent the mature miRNAs and the mi $R^{*}$, respectively

current version of the genome and the remaining seven were more precisely defined in our study. Four of the seven non-identified miRNAs have been detected in the libraries but we designated other more abundant isoforms or novel miRNAs as mature miRNAs in the corresponding precursors. The 3 other miRNAs were not identified by miRDeep-P because of potential splicing events in the hairpin sequence or because of a nonconventional folding of the stem-loop. In contrast, our study revealed 4 already identified mature miRNAs in the miRBase catalog of other species and 34 new isomiRs in addition to the set proposed by Peláez et al. Concerning the novel miRNAs, none of those identified by Peláez et al. fulfilled our selection criteria. We identified only one isomiR of a novel miRNA previously described by Peláez et al., 21 could not be mapped to the genome and 17 were present in more than 40 locations in the genome and thus were not further considered (see methods). The other 19 miRNAs were not selected because they do not fulfill our folding, splicing or expression criteria. Conversely, the availability of genomic sequence data allowed us to identify 64 high-confidence novel miRNAs that satisfy all our criteria and all those currently accepted for microRNA annotation [48]. Means of 2.5 and 6.9 hits in genome for each known and novel miRNA were found, respectively. We can ascribe this difference to a lower selection pressure on the novel miRNAs [49], compared to conserved ones, and the fact that most of these newly identified miRNAs are young miRNAs and, perhaps, their selection process is still occurring.

In summary, the new whole-genome investigation of the miRNA candidates allowed us to identify 307 genuine precursors of already known and new miRNAs and 121 high confidence unpublished mature sequences.

\section{MiRNA precursor genomic localization}

In plants, most of the miRNA genes are encoded in intergenic regions $[16,50]$ but some are present in introns, exons, or UTRs [51]. In our study, we localized the different precursors of miRNAs in the genome and determined their position relative to annotated genes. As expected, about $90 \%$ of the known and new isomiR are located in intergenic regions (Table 2). One of the known miRNAs, pvu-miR1514a, has a precursor encoded in an intron and two, bna-miR167d and gmamiR167a, are located in 3'UTRs. There are 11 other known miRNAs located in exons. The proportion of known miRNAs located in exons of annotated genes is high compared to that of miRNAs present in other gene locations; however, due to the lack of curation in the current annotation of the $P$. vulgaris genome this could be an overestimation. These sequences are located in exons of putative small proteins lacking known domains or homologs in other organisms and it is very likely that these precursors are, in fact, intergenic. For the novel miRNAs compared to known ones and new isomiRs, we encountered fewer precursors in 
intergenic regions $(\sim 80 \%$, Table 2$)$ but more in introns $(\sim 16 \%)$. Since we found a higher proportion of novel miRNAs in introns, we can imagine that some of the younger miRNA genes could originate from introns. There are different hypotheses on the origin of the miRNAs: novel miRNA genes may originate from duplication of other miRNAs, from inverted terminal repeats of transposable elements or from random stem-loop structures emerging from intergenic or intronic regions (see Zhuo et al. for review [50]). In this sense, the birth of a miRNA gene is less costly if it derives from a ready-to-use transcription unit that an intron can confer indirectly [52]. In our results, 15 of the 16 precursors of novel miRNAs identified in introns are located in the same orientation as the corresponding gene, supporting this hypothesis. On the one hand, the expression of various miRNAs located in introns is dependent on the expression of the corresponding host gene [53] and we can think that the evolutionary selection of this type of transcription mechanism is fixed. On the other hand, we can hypothesize that some of the miRNAs present in introns are young miRNAs and the use of the transcriptional unit of the host gene is a first step in the evolution and, thereafter, they may acquire their own transcriptional unit and evolve as canonical independent miRNAs thanks to duplication events or exon shuffling [54].

\section{Conservation of the identified miRNAs in plants}

We investigated the conservation of the identified miRNAs in 7 vascular plants and 1 moss. We chose two legumes (Medicago truncatula and Glycine max), three non-legume eudicots (Vitis vinifera, Populus trichocarpa and Arabidopsis thaliana), two monocots (Oryza sativa and Zea mays) and the moss Physcomitrella patens. A miRNA is considered as conserved in a given species when it is present in its full length without any mismatches. As expected, in a global view, the known miRNAs are the most conserved with 4 miRNAs (ath-miR156j, gmamiR156a, gma-miR160a and pvu-miR166a) present in all the selected genomes. We found 21 miRNAs, including 19 known and 2 new isomiRs (pvu-miR319c), conserved in all the plants except the moss (Fig. 3). A total of 118 miRNAs seem to be legume-specific: $22 \%$ of the known miRNAs, $73 \%$ of the new isomiRs and $100 \%$ of the novel miRNAs. Among them, 77 are specific to $P$. vulgaris: 1 known miRNA (pvu-miR1514a), 20 new isomiRs and 56 novel miRNAs.

The proportion of species-specific miRNAs is variable in plants. For example, in Arabidopsis thaliana and A. lyrata, $35 \%$ of the identified miRNAs are speciesspecific [55]. This is also the case of $23 \%$ of the Medicago truncatula miRNAs [44], $37 \%$ in Populus species
[56], $41 \%$ in wheat [57] and $56 \%$ in apple [58]. In our results we found that $P$. vulgaris is in the range of these other plants with around $42 \%$ of the miRNAs specific to the common bean. Naturally, these numbers may fluctuate depending on the depth of sequence obtained and the methods used for the identification and the prediction of the microRNAs. The lack of conservation for the specific miRNAs suggests that they emerged recently during evolution and can thus be considered as young miRNAs.

\section{Prediction and identification of miRNAs targets}

Using degradome data and the prediction of putative targets, we are able to present a set of targets for the identified miRNAs. The degradome data reported here were obtained using a method based on the 5'-rapid amplification of cDNA ends ( $\left.5^{\prime} \mathrm{RACE}\right)$ and further adapted for high-throughput sequencing. It allows the experimental identification of the target cleavage sites associated with miRNA cleavage on a genomic scale [59]. The regulation performed by miRNAs does not necessary include a cleavage of the target transcript. To complete the degradome data, we used a target prediction tool, called psRNATarget [60], based on reverse complementary matching between miRNA and target transcript. A mean number of one degradome target and 6.5 predicted targets per miRNA candidate were found (Additional file 3: Table S3). The known miRNAs possess significantly more degradome targets (1.9/miRNA) compared to the novel ones $(0.3 / \mathrm{miRNA})$ and also have more predicted targets (8.8/known miRNA vs. 5.8/novel miRNA). Several of the target transcripts identified here for known miRNAs correspond to previously defined targets found in other plant species, thus validating our analysis. As discussed in previous studies, the lack of conventional targets for the young miRNAs is not unusual. Most of the young miRNAs, in contrast to the conserved ones, are not involved in complex regulatory networks [41] and their evolution has sometimes been considered as neutral [49]. However, the experimental demonstration of an interaction with its predicted target is considered as "the most powerful method of validating a predicted miRNA" [61]. Although $73 \%$ of the conserved miRNAs exhibit a degradome target in our data, only around $28 \%$ of the novel miRNAs also have a target identified by degradome analysis (Fig. 4). Here we found that a significant portion of novel miRNAs has functional evidence, as revealed by degradome data, for a role in regulating gene expression, thus suggesting their relevance in different biological processes. Their degradome targets are for the most part involved in metabolic processes, biosynthesis, binding processes and various functions (Fig. 5). Compared to the conserved miRNAs that preferentially target genes involved in complex 

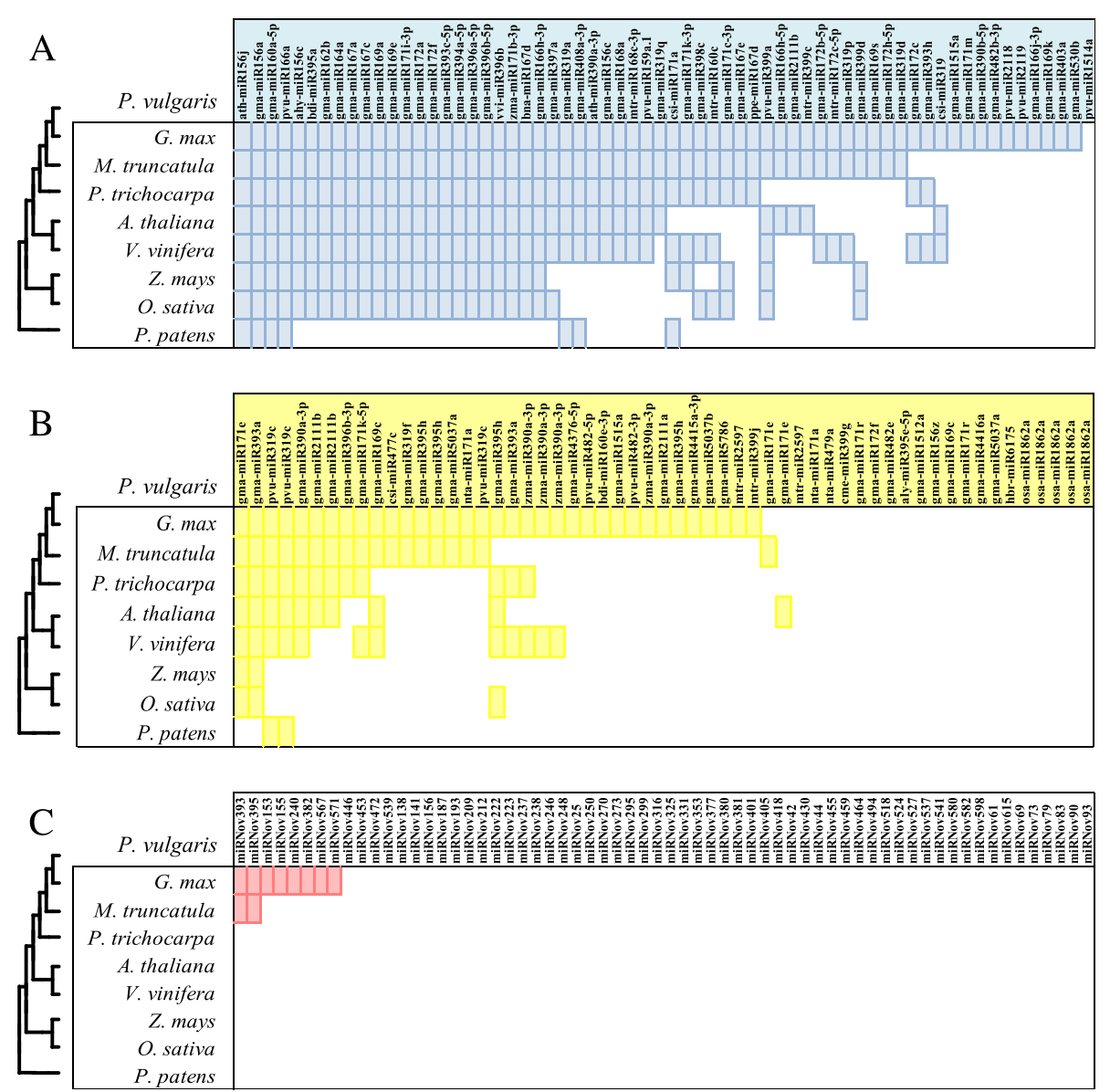

Fig. 3 Conservation of the Phaseolus vulgaris mature miRNAs in 8 plant species. The different mature miRNAs studied are listed on the first row of the tables. The first column indicates the organisms where the sequences have been searched for and the other columns correspond to the different $\mathbf{a}$ known miRNAs, $\mathbf{b}$ new isomiRs and $\mathbf{c}$ novel miRNAs. The presence of a colored square at the intersections between rows and columns indicates the $100 \%$-homology presence of the corresponding mature sequence in the genome of the corresponding species. The tree on the left indicates the phylogenetic relationship between the studied species

regulatory networks such as transcription factors [62], young miRNAs tend to target more precise and diversified functions. However, one of them, miRNov138, seems to target a transcript coding for a Homeoboxleucine zipper family protein. This transcription factor family is known to play an important role during the growth and development of plants by modulating phytohormone-signaling networks [63] and also in the interaction with microorganisms, especially during nodulation [64]. This young miRNA is specific to $P$. vulgaris and we hypothesize that it has been selected to perform a more precise role in common bean, like adaptation to a specific environment [63] or interaction with Rhizobium.

\section{Analysis of microRNA co-expression networks in nodules}

To understand the role of the newly identified miRNAs in nodules, we constructed weighted correlation networks of miRNAs. These networks describe the pairwise relationship among miRNAs that differentiate the nodule library from other libraries based on the miRNA expression patterns [65]. Using this approach, we identified 2 networks including pairwise relationships between novel miRNAs, new isomiRs and conserved miRNAs.

One of these networks is composed by 4 novel miRNAs (miRNov153, miRNov155, miR156 and miRNov494), two new members of the miR399 family, and gma-miR319d suggesting that they could act jointly (Fig. 6A). All these miRNAs have the same expression pattern, with increased expression in nodules (Additional file 1: Table S1). As shown in Fig. 7A, increased expression of pre-miR319d, pre-miRNov153/155 and pre-miRNov494 in nodules was experimentally validated by qRT-PCR. More precisely, the new isoform of the mtr-miR399j was specifically identified in nodule (Additional file 2: Table S2). This miRNA family is involved in phosphate homeostasis [66] but, furthermore, has been identified as being repressed by $\mathrm{N}$-starvation [67]. Nodulation efficiency and functionality 
A
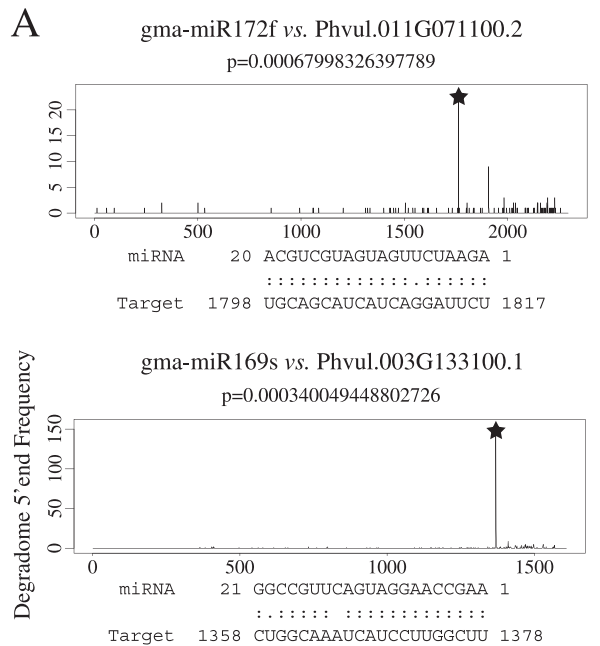

gma-miR319a vs. Phvul.001G101000.2 $\mathrm{p}=0.00339529569051544$

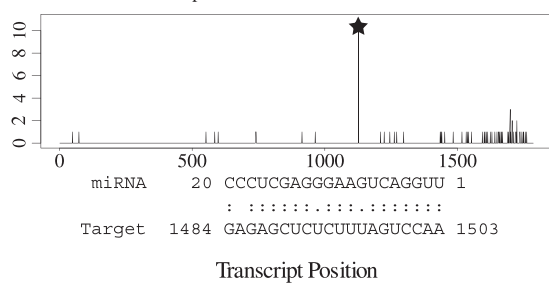

B miRNov42 vs. Phvul.004G172700.1 $\mathrm{p}=0.033438864562926$

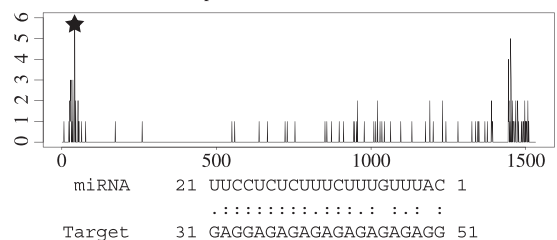

miRNov395 vs. Phvul.009G172100.1 $\mathrm{p}=0.0255169494760313$

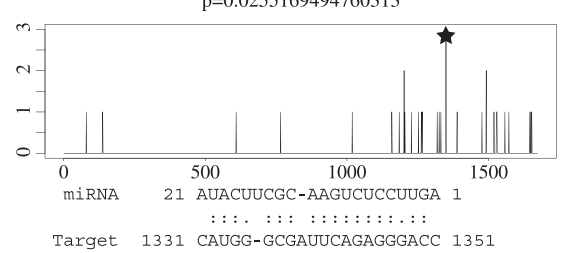

miRNov446 vs. Phvul.004G002700.1 $\mathrm{p}=0.0218887954277773$

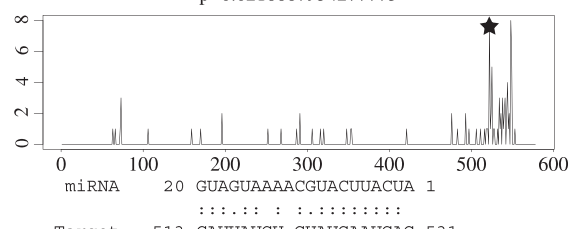

Target 513 CAUUAUGU-GUAUGAAUGAG 531

Transcript Position

Fig. 4 Target plots of miRNA-targeted transcripts using degradome data. Target plots of the distribution of degradome read abundance on transcripts targeted by $\mathbf{a}$. known miRNAs and $\mathbf{b}$. novel miRNAs. Vertical axes display the read abundance and the horizontal axes display the precursor position by base pair. The black vertical bars show the read abundance for the corresponding precursor base. The black stars indicate signatures consistent with the miRNA-directed cleavage. The corresponding $p$-values are shown above the plots

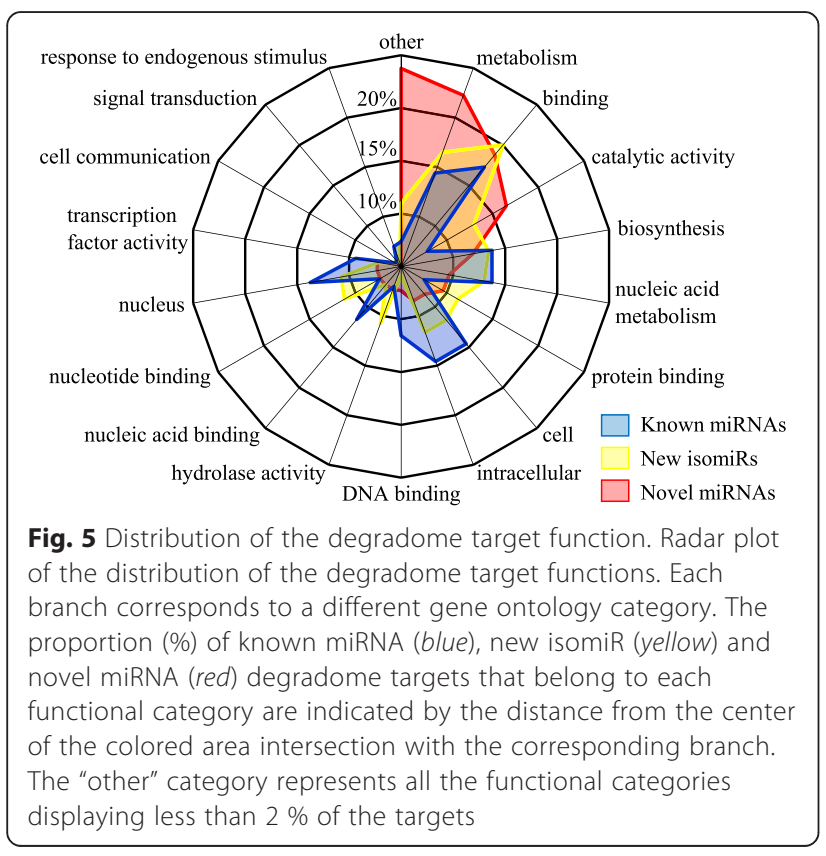

are related to these two nutrients $[68,69]$ and we hypothesize that this new isoform of miR399 could indirectly regulate the normal establishment of nodulation in $P$. vulgaris. Indeed, this miRNA family is known to target a transcript encoding $\mathrm{PHO} 2$, an ubiquitin-conjugating enzyme crucial for acquisition and translocation of phosphate [70]. In our data, we predicted another target for the new isoform of miR399 which could regulate a NB-ARC domain-containing protein, one of several factors known to be involved in the plant resistance and activation of innate immune responses [71]. The organ-specific accumulation of this miRNA and the function of the corresponding target suggest a role in the regulation of nodule-specific defense mechanisms. Two of the novel miRNAs from this network; miRNov153 and miRNov494, are specific to the nodule library. The miRNov494 is predicted to target two transcripts in our degradome data (Additional file 3: Table S3). qRT-PCR expression analysis showed that in nodules the expression of miRNov494 precursor increased while the expression of one of the predicted targets, an aldehyde dehydrogenase (Phvul.004G162200.1) decreased (Fig. 7A). The members of this protein family are known to change their expression in response to a wide variety of stresses and are important in supporting environmental adaptability 

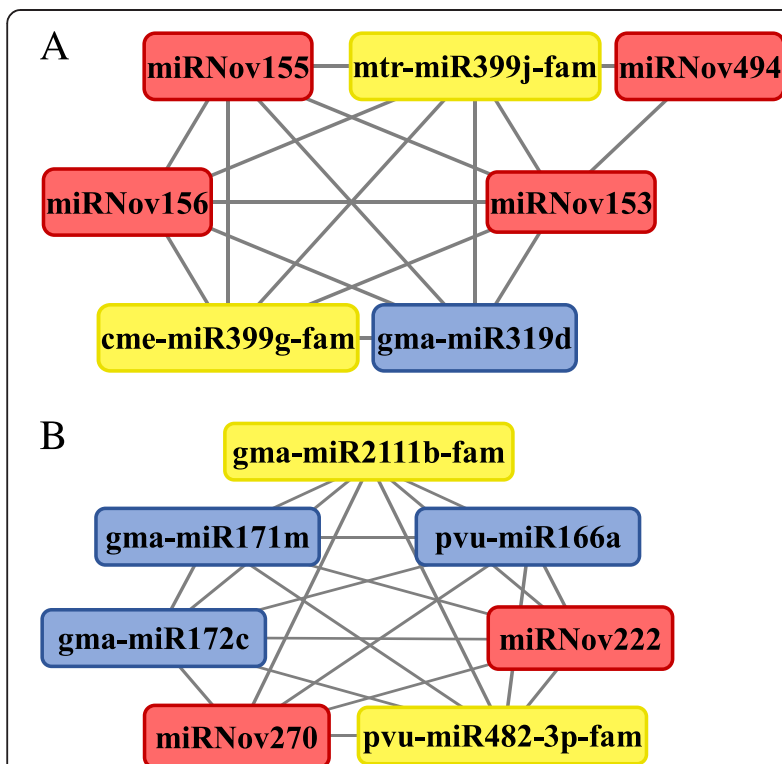

Fig. 6 Weighted correlation network analysis of microRNAs from nodules versus other organs. Representation of two regulatory networks $\mathbf{a} \& \mathbf{b}$ between the miRNAs. The nodes correspond to each miRNA encountered in the corresponding network. The edges correspond to the direct connection and relation between the nodes. Blue, yellow and red tags indicate the known miRNAs, new isomiRs and novel miRNAs, respectively

[72]. Our data suggest that the miRNov494 can regulate a member of the aldehyde dehydrogenase family in nodules and may help the plant to control the life cycle of this symbiotic organ. MiRNov153 is $\sim 18$ times more highly expressed than the miRNov494 and is encoded in an intron of an F-box protein gene (Additional file 1: Table S1). Part of this protein family plays important role in root symbioses [73] and particularly in the auto-regulation of nodulation [74]. This novel miRNA, miRNov153, is predicted to target an uridine kinase (Phvul.003 g180800), for which no expression variation has been measured in nodule compared to root tissue, and a transcript coding for a hypothetical protein (Phvul.002 g255600), for which we have observed an expression inversely correlated to its regulator miRNA in nodule (Fig. 7A). Although this target does not guide us to an obvious functionality, the fact that this miRNA is encoded in an intron of an F-box protein gene, in the same orientation, would permit us to envisage that this miRNA is co-expressed with the host gene and could act in the regulation of the systemic negative feedback control of nodulation. Among the 8 species for which we searched for the presence of the miRNov153, only the soybean genome encodes this miRNA and displays a bona fide hairpin-like precursor (Additional file 4: Figure S1A). To check if this locus can produce a miRNA corresponding to the mature miRNov153 in soybean, we mapped public nodule small RNA sequences from Arikit et al. [75] to the miRNov153 soybean precursor (Additional file 4: Figure S1B). Tens of sequences mapped exactly to miRNov153 and the corresponding miRNov153* and only three sequences mapped to other coordinates, suggesting that nodules from soybean also produce the miRNov153. The available public small RNA libraries from nodule represent 5 time points from 10 dpi to $30 \mathrm{dpi}$. In these libraries, we observe an increased expression of the miRNov153 during nodule development. Additionally, this miRNA is absent from Medicago truncatula, which develops indeterminate nodules, and is conserved in common bean and soybean, two species developing determinate nodules. These results tend to show a role of miRNov153, via its targets, in the loss of meristematic activity or the senescence of determinate nodules.

To our knowledge, no link has been published before between miR319 and nodulation. This miRNA is a regulator of the plant stress responses against salinity, drought or cold [76, 77] but it is also implicated in the regulation of cell proliferation via the control of its targets, members of the TCP transcription factor family [78]. Here, we have validated the increase of miR319d precursor expression in nodules and the decrease of one of its predicted targets, a TCP transcription factor family member (Phvul.011 g156900), in the same tissue (Fig. 7A). Because this miRNA is present in a network that distinguishes the nodule library from the others, and because it is connected with miRNAs described in previous studies as being related to nodules, we hypothesize that miR319 and its targets are strong candidates for which a role in nodule development must be investigated.

We identified a second network involving 7 miRNAs: 2 novel ones, 2 new isomiRs and 3 already known miRNAs (Fig. 6B). In this network, we retrieved members of miRNA families already described as regulating nodule development. Specific variants of $L$. japonicus and $M$. truncatula miR171 target the GRAS-family NSP2 TF, a key regulator of the common symbiotic pathway for rhizobial and arbuscular mychorrizal symbioses [79-81]. MiR166 and its target gene, a HD-ZIP III transcription factor, regulate meristem activity and vascular differentiation in roots and nodules [64]. In soybean, the overexpression of miR482, targeting a GSK-3-like protein MsK4, resulted in an increase in nodule number without affecting root development or the number of nodule primordia [36]. The crucial role of miR172 and its target gene, a transcription factor from the AP2 family, have been described for soybean- and common bean-rhizobia symbiosis [82-84]. For soybean, Yan et al. [82] postulated that miR172 regulation of nodulation is explained by the AP2 repression of non-symbiotic hemoglobin $(H b)$ gene expression that regulates the level of nodulation; while Wang et al. [84] recently reported that soybean NNC1, a AP2 transcription factor target of 


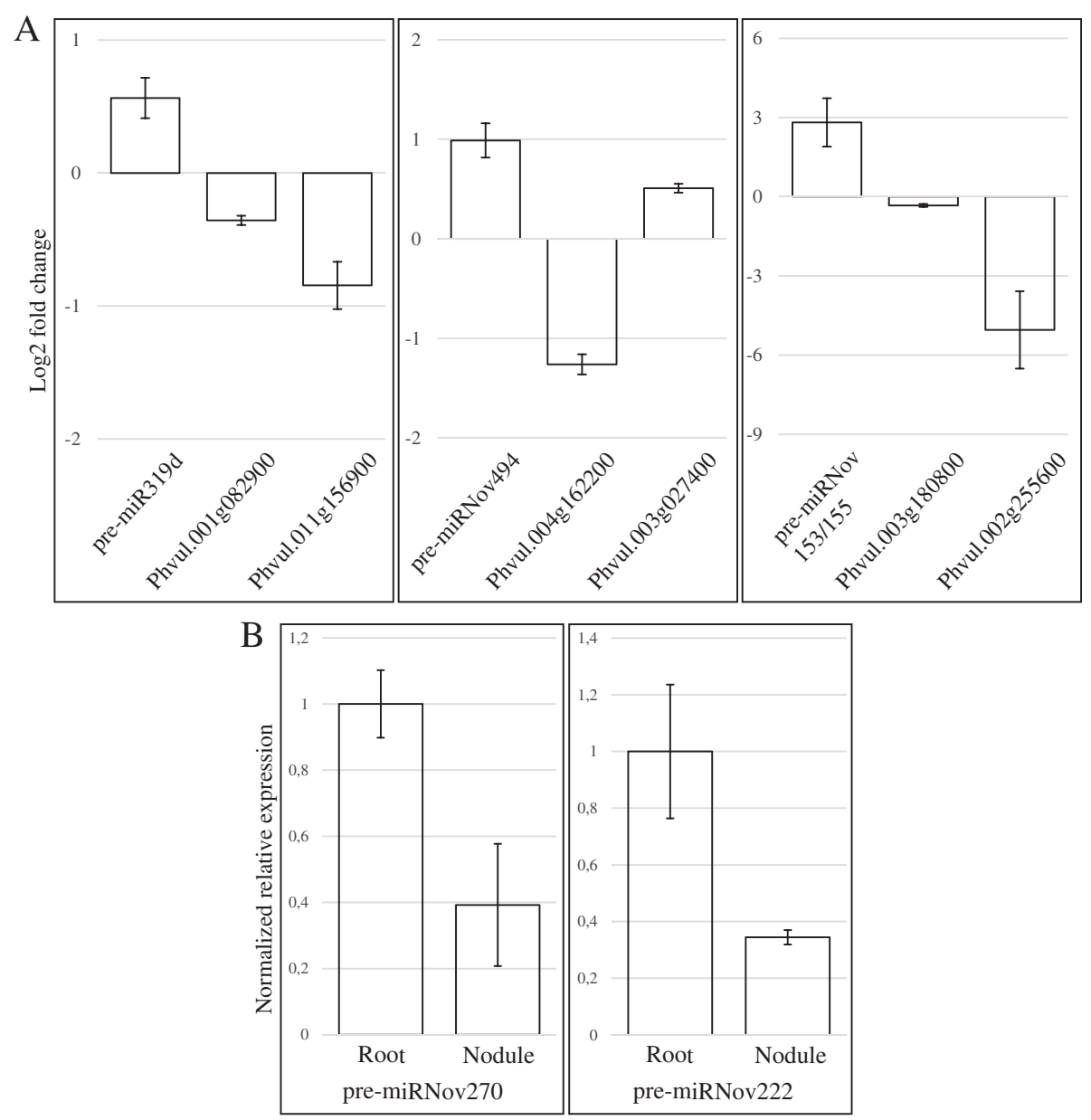

Fig. 7 Expression quantification of miRNA precursors and their targets involved in the weighted correlation networks. Relative quantification by qRT-PCR of miRNA precursors and their corresponding targets. a Expression of miRNAs and corresponding targets in nodules compared to root tissues from the Fig. 6A network. Histograms represent the Log2 fold change level and error bars represent the standard error of the mean of the three expression ratios from three biological replicates. $\mathbf{b}$ Expression of novel miRNA precursors from Fig. 6B network. Histograms represent the relative expression and error bars represent the standard error of the mean of the three relative expressions from three biological replicates. Expression values have been standardized at 1 for the control roots and proportionally adjusted for nodule expression. The geometric mean of the expression of three housekeeping genes was used for normalization of the expression levels

miR172, represses ENOD40 expression that results in negative regulation of early symbiotic stages. For common bean, we recently demonstrated [83] that miR172c, which has the transcription factor AP2-1 as target gene, indirectly regulates the expression of the transcription factors NF-YA1, NSP2 and CYCLOPS as well as the gene FLOT2, all of which are essential regulators of early stages of the symbiosis. In addition, we postulated that miR172-induced AP2-1 silencing in mature common bean nodules is involved in downregulating expression of genes related to nodule senescence postulated as targets of AP2-1 transcription activation [83]. We also found a new isomiR of a family already described as regulating the development of nodules: the miR2111 targeting a Kelch-related proteins [85]. Finally, the last two miRNAs encountered in this network are new miRNAs: miRNov222 and miRNov270. Although these miRNAs have low expression and present no processing evidences in degradome data or transcription evidences in transcriptome data (Additional file 1: Table S1), we have detected the corresponding precursors by qRT-PCR in roots and confirmed their expression decrease in nodules (Fig. 7B). As elements in a network containing miRNAs previously described as nodulation regulators, these novel miRNAs are also probably involved in the normal establishment and functioning of the nodulation process via regulation of their targets. These two miRNAs putatively target a protein kinase and a NB-ARC domain-containing disease resistance protein, respectively (Additional file 3: Table S3). Although the protein kinases belong to a large protein family, various members are known to be involved in signalling during 
nodulation [86] and, as described above for the putative target of the new isoform of the miR399, the NB-ARC domain-containing disease resistance proteins are known to be involved in the plant resistance and activation of innate immune responses [71] and could play a specific role in the nodule and allow the proper functioning of the nitrogen-fixing organ. These novel miRNAs are directly related to regulators of nodulation and it is conceivable that these Phaseolus-specific miRNAs could act in the same way as the connected conserved miRNAs and play a role during nodule establishment in a more spatio-temporal specific manner. Like the miR319 family, these novel miRNAs must be considered as major candidates for deciphering the specific regulation of nodulation in $P$. vulgaris.

\section{Identification of phasi-RNAs and their associated targets}

In all the sequenced libraries, a large number of reads do not correspond to miRNAs or other previously identified non-coding RNAs of common bean, suggesting they could belong to another class of small RNAs. We decided to focus on phasiRNAs and, based on the alignment of the reads with the genome and the phasing score for each locus, we predicted 125 statistically significant loci producing 21 nt-phased small RNAs (Additional file 5: Table S4, see example in Fig. 8A). Among the identified PHAS genes, only two are conserved in the three legumes studied, i.e. M. truncatula, G. max and $P$. vulgaris (Additional file 6: Figure S2). One of these PHAS genes is known as the TAS3 gene targeted by the miR390 and producing small RNAs targeting transcripts that encode AUXIN RESPONSE FACTORs (ARF3 and ARF4) [87]. In our data, we also identified miR390 and its regulatory action on the TAS3 gene (Additional file 5: Table S4), providing a positive control for our methodology. The second PHAS gene codes for a member of the disease resistance protein (TIR-NBS-LRR class) family known to be targeted by miR2118 in soybean but not yet in $M$. truncatula [71]. In our analysis, we encountered that miR2118 potentially targets this PHAS gene but not in the expected phase. However, for this gene, we have identified a potential in-phase slicing by a novel miRNA, the miRNov212 (Additional file 5: Table S4). Among the 125 loci identified, 47 are predicted to be targeted by 31 different miRNAs with a cleavage site in phase with the initiation site of the corresponding phasiRNAs. In recent years, loci producing 21 ntphasiRNAs (PHAS genes) have been identified in different species and their number in each case varies widely: 50 in Arabidopsis, 114 in M. truncatula, 157 in apple, 353 in peach and 864 loci in rice [40,71,88-90]. Not all PHAS genes are associated to a miRNA triggering phased small RNA, and the proportion of PHAS genes targeted by a miRNA also varies from species to species. For example, 26

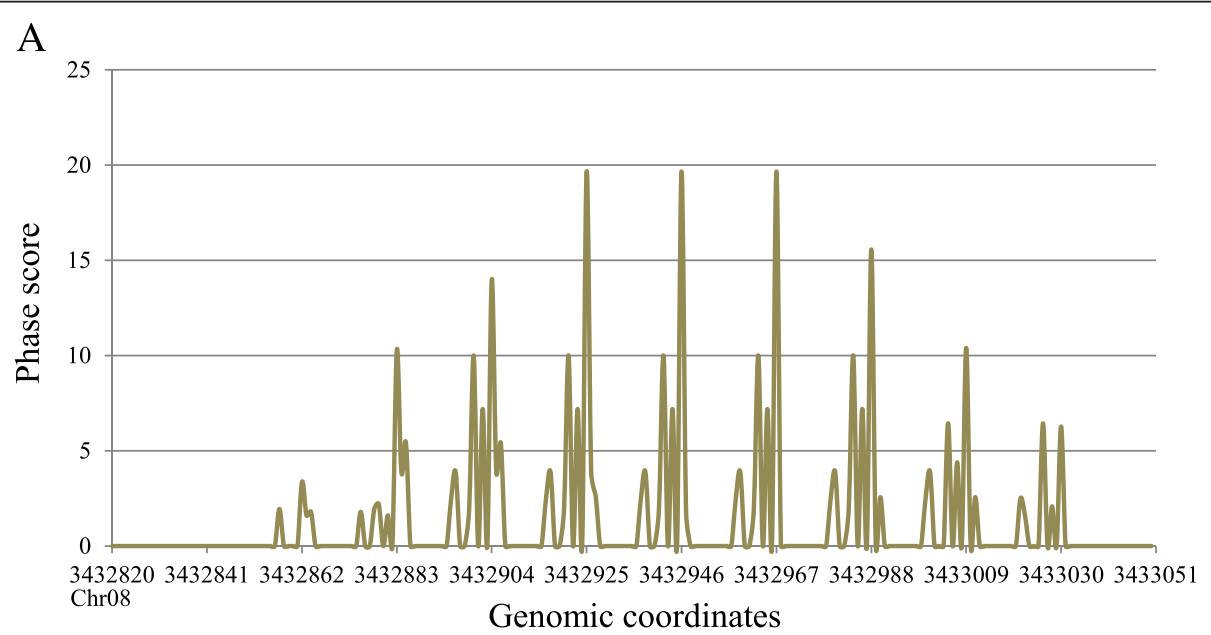

B

miRNov582

22 CCGGAGTTTCAGGTGAGGAGG 1

GGACAAGAGTGTCAAAAAGATGACTCTTCTTATTTCTATTGTATAGTGTAGGGGTGCGATATTAAATAAATA GGTGTGAATGTATTAGAGAGAGTCACATTGTCCATGTGACTTAGGAGAGTAAGTGTAGGTGTAGTTTTTAG GATGTGTCATAGTAGAAAAAGGTCACACCTCCCATGTGACTTGTTTTTTGTGGGAATTTCAAATGAGTTTC ATTTGAATTTCCCACCTATTTTCAGCACCCTCACACTATAAATAGAGGTGTGgGCTCTTGTAAAATTCA GAATTGGAAATTGGAT

Chr08:3432925-3433198

Fig. 8 Identification of a phasiRNA loci and their triggering miRNAs. a Distribution of the phase score (vertical axis) as a function of the genomic coordinates (horizontal axis) of the PHAS gene Chr08:3432925-3433198 coding for a pfkB-like carbohydrate kinase family protein. b Representation of the miRNov582 triggering the $21 \mathrm{nt}$ phasiRNA production (green and yellow alternation) of the PHAS gene Chr08:3432925-3433198. The G-U hydrogen bonds are represented by a single dot and the $\mathrm{A}-\mathrm{U}$ and $\mathrm{G}-\mathrm{C}$ hydrogen bonds by two dots 
of the 157 apple PHAS genes and 160 of the 864 rice PHAS genes are potentially targeted by miRNAs that can trigger phasiRNA production. Here, we identified 13 known miRNAs, 4 new isomiRs and 14 novel miRNAs that potentially trigger the production of phasiRNAs (an example is shown in Fig. 8B). Most of the 17 previously identified miRNAs are known to be involved in the production of phasiRNAs in other species [27, 91]. The phasiRNAs produced by these known miRNAs originate from transcripts encoding proteins involved in a wide range of functions like the MYB genes targeted by miR159, NB-LRR genes targeted by miR482 and miR2118, $\mathrm{Ca}^{2+}$-ATPases targeted by miR4376 or TIR/AFB targeted by miR393 [27]. Uncharacteristically, we identified one PHAS gene (Chr11: 2402498-2402857) that possesses all the features to produce two sets of phasiRNAs derived from two distinct phases that are triggered by two novel miRNAs (miRNov141 and miRNov316). The resulting phasiRNAs are only expressed in the flower and the corresponding miRNAs are almost uniquely expressed in the same organ (Additional file 2: Table S2). These phasiRNAs are produced from a transcript coding for a putative serine carboxypeptidase-like 35, a member of a large family involved in multiple cellular processes. In rice, a member of this family has been identified as playing a role in defense against pathogens and oxidative stress [92]. The mechanism of double-phasing by two different miRNAs allows production of two overlapping sets of phasiRNAs from the same sequence and we can imagine this confers an additional layer of small RNA production allowing the more precise regulation of defense reactions of the organism. The complete set of PHAS genes targeted by microRNAs must be larger than the number reported here, since we may not have a complete list of miRNAs yet. In addition, other miRNAs may generate phasiRNAs by cleaving target transcripts at an outof-phase position by a phenomenon called phase-drift, caused by a DCL slippage [93], and, identification of these cleavage events becomes challenging.

All of the studied organs presented transcripts producing 21-nt phasiRNAs but only 13 of the 125 PHAS genes $(\sim 10 \%)$ were identified in the five different small RNA libraries (Additional file 7: Figure S3). Moreover, most of the phasiRNAs are organ-specific $(\sim 73 \%)$ and flowers present the largest set of identified phasiRNAs expressed, with around $73 \%$ of the loci, including 69\% of organ-specific expressed loci. Conversely, nodules had lower expressed phasiRNAs, with $\sim 13 \%$ of the identified loci. 86 of the 125 PHAS loci are localized in a predicted transcript, including 46 with an associated putative function (Additional file 5: Table S4) suggesting that the corresponding phasiRNAs can also target these transcripts or other members of the corresponding gene families.
Among the phasiRNA loci localized in transcripts with a putative function, two are expressed in all the organs and are derived from an Auxin signaling F-box 2 and a NAC transcription factor-like 9. These two proteins are related to auxin signaling and lateral root formation [94] and the corresponding families are known to be common targets of phasiRNAs [40]. The presence of the corresponding phasiRNAs in all the studied organs reflects the important role in regulation of these small RNAs at the organism level. MiR2118 is predicted to target two PHAS genes (Chr04:3128306-3129229 and Chr04: 9380782-9381299) coding for proteins involved in disease resistance: a NB-ARC domain-containing disease resistance protein and a Disease resistance protein (TIR-NBS-LRR class) family member. These loci are expressed in all the organs except nodules (Additional file 5: Table S4). Although miR2118 has been retrieved in all the organs studied, including nodules, we can hypothesize that miR2118 regulates nodule growth via the control of phasiRNA production derived from the NB-ARC and TIR-NBS-LRR transcripts. The investigation of phasiRNAs is quite recent and we envisage that, in coming years, the number of PHAS genes will increase, as it has for miRNAs in recent years, in terms of loci per species and number of studied plant species.

\section{Conclusions}

Thanks to the genome-wide identification of miRNAs and phasiRNAs from $P$. vulgaris, we provide a set of sequences allowing the extension of the sRNAome of common bean. The investigation of the miRNA degradome targets, the miRNA correlation networks and the PHAS gene function permitted us to propose a role for the identified small RNAs and provide genuine sequence candidates to be studied in plant-microorganism interactions and specifically in the root-nodule symbiosis.

\section{Methods \\ Plant materials and sequencing \\ Plant materials}

Flower, leaf, root and seedling raw sequences were obtained from the study of Peláez et al. [29]. Briefly, Phaseolus vulgaris L. cv. Negro Jamapa and cv. Pinto Villa were grown and roots ( 15 day old) and flower buds (35-40 day old) were collected in liquid $\mathrm{N}_{2}$ and stored at $-80^{\circ} \mathrm{C}$. Whole 14 days old seedlings were collected in liquid $\mathrm{N}_{2}$ and stored at $-80{ }^{\circ} \mathrm{C}$. A pool of leaves from 10 and 20 day-old plants was harvested for RNA purification.

\section{Nodulation conditions}

P. vulgaris L. cv. Negro Jamapa seeds were surface sterilized and germinated under sterile conditions for two days and then planted in pots with sterile vermiculite. A 
fresh inoculum of $R$. tropici CIAT899 grown on PY liquid medium supplemented with $\mathrm{CaCl}_{2}(7 \mu \mathrm{M})$, rifampicin $(50 \mu \mathrm{g} / \mathrm{ml})$, and nalidixic acid $(20 \mu \mathrm{g} / \mathrm{ml})$ at $30{ }^{\circ} \mathrm{C}$ to a cell density of $5 \times 10^{8} \mathrm{ml}^{-1}$ was prepared. Immediately after transferring bean plants into pots with fresh sterile vermiculite, each plant was inoculated by adding $1 \mathrm{ml}$ of the $R$. tropici culture directly to the root. Plants were grown in a greenhouse with a controlled environment $\left(25-28{ }^{\circ} \mathrm{C}, 16 \mathrm{~h}\right.$ light $/ 8 \mathrm{~h}$ dark) and were watered with nitrogen-free B\&D nutrient solution [95] every 2 days. Root nodules were collected 18 and 27 days after inoculation.

\section{Library preparation and sequencing}

Total RNA was isolated from frozen samples using the Trizol reagent according to the manufacturer's instructions (Invitrogen, Carlsbad, CA) and $10 \mu \mathrm{g}$ of each sample was prepared for Deep Sequencing following Illumina's Small RNA alternative sample preparation protocol v1.5. Complementary DNA (cDNA) libraries were separately prepared and Single Read-sequenced using the Genome Analyzer IIx (GAIIx) (36 bp) and the Illumina Cluster Station (Illumina Inc, USA) at the Instituto de Biotecnología (Universidad Nacional Autónoma de México) and raw reads from the Illumina Pipeline 1.4 for the small RNA libraries were purged of sequence adapters, low quality tags and small sequences $(<16 \mathrm{nt}$ long) [29]. The contamination of the nodule library by bacterial sequences was investigated by BLASTn of the whole nodule small RNA set against the $R$. tropici CIAT899 genome as a reference. Raw reads for the small RNA sequencing are available in the Gene Expression Omnibus database under accession number GSE67409.

\section{Degradome library construction Plant material}

Seeds of P. vulgaris, var. Pinto Villa were surface sterilized in $50 \%(\mathrm{v} / \mathrm{v})$ sodium hypochlorite and $0.5 \%(\mathrm{v} / \mathrm{v})$ Tween-20 for $3 \mathrm{~min}$, rinsed with distilled water for $10 \mathrm{~min}$ and germinated in wet paper towels in the dark at $24{ }^{\circ} \mathrm{C}$ for 3 days. At this point seedlings of similar size were transferred to moist vermiculite watered to substrate capacity and maintained at $24{ }^{\circ} \mathrm{C}$ for $48 \mathrm{~h}$ with $16 \mathrm{~h} / 8 \mathrm{~h}$ light/dark day cycles. Seedlings were collected, ground to a fine powder under liquid $\mathrm{N}_{2}$ and preserved at $-80{ }^{\circ} \mathrm{C}$ until the material was used for RNA preparation.

\section{Library construction for high throughput sequencing}

The protocol used to obtain cDNA libraries for degradome analysis was carried out essentially as previously described [59, 96]. Total RNA from seedlings was obtained using the Trizol reagent (Life Technologies) according to the manufacturer's directions. Subsequently,
poly-A+ RNA was ligated to a 5'adapter containing a MmeI site at its 3'-end. The ligated products were used for cDNA production and amplified by PCR for five cycles. The PCR products were digested with MmeI and the resulting fragments ligated to a second doublestranded oligonucleotide, purified and amplified for another ten PCR cycles. The final product was purified and subjected to high throughput sequencing as described below.

\section{High Throughput sequencing}

DNA libraries were subjected to Single Read-sequencing (36 bp) using a Genome Analyzer IIx (GAIIx) and the Illumina Cluster Station (Illumina Inc, USA) at the UNAM Sequencing facility (Unidad Universitaria de Secuenciación Masiva de DNA, Universidad Nacional Autónoma de México). Raw reads for the degradome sequencing are available in the Gene Expression Omnibus database under accession number GSE67432.

\section{Bioinformatics analysis of sequencing data miRNA identification}

The identification of the miRNA precursors was performed using the miRDeep-P pipeline [33] with a maximal identification window size of $250 \mathrm{nt}$, no mismatch allowed and a number of hits in the genome lower than 40. The precursors with a small RNA overlapping an ncRNA (tRNAs, rRNAs and other ncRNAs) were discarded by the software. We recovered only the precursors with a size of mature miRNA between $18 \mathrm{nt}$ and $25 \mathrm{nt}$ wherein the mature miRNA lies within the top $5 \%$ of the most expressed sequences, in a given library. The selected mature miRNAs were compared with all the Viridiplantae miRNAs of the miRBase version 21 [28] using the NCBI BLASTn program [97], allowing no mismatches to identify the already known miRNAs. To identify the miRNAs families and the new isoforms of already known miRNAs, we used CD-HIT (Cluster Database at High Identity with Tolerance) [98] with at least $84.2 \%$ of identity. For the novel miRNAs, a more stringent selection was performed and only those mature sequences for which the best precursor miRDeep score is greater than 2.2 (the threshold for the top $5 \%$ of the precursor miRDeep scores) have been kept. To list all the precursors encoding for the final set of novel genuine mature miRNAs, we recovered all the corresponding precursors that have been identified by miRDeep-P and passed all our filters without a selection on the attributed score.

\section{Target identification}

Two programs were used to identify the targets of the selected miRNAs. CleaveLand ver.4 [99] was used to identify the putative target sites from degradome data 
(see Degradome library construction section). The transcripts from the version 1 of the $P$. vulgaris genome [2] were used as target templates, the total set of identified miRNAs has been used as the small RNA candidates and only targets with a p-value lower or equal to 0.05 were selected. psRNATarget [60] was used to predict putative miRNA targets on the same transcript dataset. Default parameters were used and only the targets with an expectation value lower than 3 were retained.

To identify the functional distribution of these sets of targets, we used the corresponding Gene Ontology Annotation [100] and classified the corresponding GO terms with CateGOrizer [101] using the GO_slim2 classification with the "accumulative all occurrences" count method.

\section{Organ distribution and conservation analyses}

The organ distribution was performed manually: a miRNA is considered as present in an organ when the corresponding mature sequence is in the top $5 \%$ of the most expressed mapped sequences of the corresponding organ library. The "five sets" Venn diagrams were inspired by Edwards' Venn diagrams [102].

Conservation of mature miRNAs was investigated using the NCBI BLASTn program [97] against the genomes of 8 species: two Fabaceae (Medicago truncatula 4.0 and Glycine $\max$ Wm82.a2.v1), three non-legume eudicots (Vitis vinifera Genoscope.12X, Populus trichocarpa 3.0 and Arabidopsis thaliana TAIR10), two monocots (Oryza sativa 7.0 and Zea mays 6a) and the moss Physcomitrella patens 3.0. No mismatches were allowed in identifying the corresponding homologous sequences.

\section{Weighted correlation network analysis of microRNAs}

The weighted correlation networks were constructed with the WGCNA package for $\mathrm{R}[65,103]$ following the automated one-step protocol and with the default parameters except the minimum module size of 3 miRNAs in order to discover the smallest networks. We used normalized expression data of the 185 mature miRNAs according to the formula: (miRNA read number * 1.000.000)/total mapped reads per library. The eigengene value was calculated for each module and networks in order to test the significant association with the nodulation trait (the difference between the nodule library and the other libraries). Then, the networks were drawn using Cytoscape [104].

\section{PhasiRNA identification}

Bowtie alignments [105] without mismatch were used to predict the phasiRNAs. Phasing score [71] was calculated for each $21 \mathrm{nt}$ read mapped to the genome with a threshold of 15 as mentioned in Zhai et al., [71]. To eliminate the contribution of random fragments generated from RNA degradation products a chi-test was performed per locus, taking into account the number of reads in phase and the reads not in phase $(p<0.01)$. Finally, a locus was predicted as a phasiRNA if it passed the above filters and had 3 or more 21 nt windows with a number of reads in the most abundant $5 \%$ and genome mapped reads in at least one library. All loci located in transposable elements were removed. Once the final phasiRNA-generating loci were determined, they were tested for the identification of phase-triggering microRNAs/phasiRNA-targeted transcripts using psRNATarget [60] with default parameters and a limit of expectation of 5 .

\section{Sample preparation and expression analysis}

Total RNA was isolated from $100 \mathrm{mg}$ of frozen roots and nodules (21 dpi) from three biological replicates using Trizol reagent (Life Technologies) following the manufacturer's instructions. cDNA was synthesized from $2 \mu \mathrm{g}$ of total RNA using RevertAid ${ }^{\mathrm{m}} \mathrm{H}$ Minus First Strand cDNA Synthesis Kit (Fermentas). Resulting cDNAs were then diluted and used to perform qRT-PCR assays using SYBR Green PCR Master Mix (Applied Biosystems), following the manufacturer's instructions. The sequences of oligonucleotide primers used are provided in Additional file 8: Table S5. Reactions were analyzed in a real-time thermocycler Applied Biosystem 7300. Two technical replicates were performed for each reaction. Relative expression was calculated with the "comparative Ct method" and normalized with the geometrical mean of three housekeeping genes (HSP, MDH \& Ubiquitin9) [106].

\section{Availability of supporting data}

The RNA-seq data sets supporting the results of this study are available in the Gene Expression Omnibus (GEO) database, under accession GSE67433.

\section{Additional files}

\footnotetext{
Additional file 1: Table S1. List of the identified microRNA precursors and their corresponding mature forms. The blue, yellow and red lines represent the known miRNAs, the new isomiRs and the novel miRNAs, respectively. The "All precursors" sheet lists all the precursors identified that fulfill our criteria. The "All matures nr" sheet lists all the non-redundant mature miRNAs resulting from the "All precursors" list. The "Families" sheet lists all the miRNA families identified in our study and the corresponding number of members found.

Additional file 2: Table S2. List of the miRNAs and the corresponding number of reads present in the different organs. The blue, yellow and red lines represent the known miRNAs, the new isomiRs and the novel miRNAs, respectively. The absence or presence of a " 1 " in the presence columns indicate the absence or the presence of the corresponding miRNA (lines) in the corresponding library (rows)

Additional file 3: Table S3. List of miRNA degradome and psRNATarget-predicted targets. The blue, yellow and red lines represent the targets of the known miRNAs, the new isomiRs and the novel miRNAs, respectively. The "Degradome targets", "Degradome targets rejected miRs", "psRNATarget-predicted targets" and "psRNATarget target reject" sheets list the
} 
targets predicted by degradome data for the selected and rejected miRNAs, and the targets predicted by psRNATarget for the selected and rejected miRNAs, respectively.

Additional file 4: Figure S1. Distribution of sequencing reads from soybean nodule libraries mapped on the soybean precursor of miRNov153. (A) Minimum free energy structure prediction of miRNov153 precursor of soybean. Heat colors represent the base-pair probabilities for each nucleotide (Blue $=0$; Red $=1$ ). The brackets show the position of miRNov153 mature and star. (B) Visualization of the mapped read distribution on the miRNov153 soybean precursor. miRNA reference sequence is at the top. The oriented grey bars represent the mapped reds. The brackets show the position of miRNov153 mature and star.

Additional file 5: Table S4. List of the identified PHAS genes and their distribution in 5 plant organs. The presence of a "0" or " 1 " in the libraries columns indicates the absence or presence of the corresponding PHAS locus (lines) in the corresponding library (rows), respectively. The conservation column indicates the initials of the legume species in which the PHAS locus is conserved (Gma = Glycine max, Mtr = Medicago truncatula).

Additional file 6: Figure S2. Venn diagram of the conservation of the PHAS genes in 3 legume species. Venn diagram of the distribution of PHAS genes based on their presence in three legumes: Medicago truncatula (red), Glycine max (yellow) and Phaseolus vulgaris (blue). The numbers in each Venn diagram area correspond to the numbers of PHAS genes encountered in the corresponding overlapping species area.

Additional file 7: Figure S3. Distribution of the identified PHAS genes in 5 plant organs. Venn diagram of the distribution of PHAS gene expression in the different studied organs. Red: flower, green: leaf, yellow: root, blue: seedling and black: nodule. The numbers in each Venn diagram area correspond to the numbers of expressed phasiRNA loci encountered in the corresponding overlapping organ area.

Additional file 8: Table S5. List of oligonucleotide sequences used in quantification experiments.

\section{Abbreviations}

5'RACE: 5'-rapid amplification of cDNA ends; AGO: Argonaute; bp: Base pair; Cv: Cultivar; dsRNA: Double-strand RNA; GO: Gene Ontology; kcal: Kilocalorie; miRNA: microRNA; mol: Molar; MYB: Myeloblastosis; NB-LRR: Nucleotide Binding domain (NB) and a Leucine Rich Repeat (LRR) domain; ncRNA: Non-coding RNA; NSP: Nodulation Signaling Pathway; nt: Nucleotide; PPR: Pentatrico peptide repeat; RISC: RNA-induced silencing complex; rRNA: Ribosomal RNA; TasiRNA: Trans-acting siRNAs; tRNA: Transfer RNA; UTR: Untranslated region.

\section{Competing interests}

The authors declare that they have no competing interests.

\section{Authors' contributions}

DF performed the miRNA analyses, supervised all the analyses, the data interpretation, and wrote the manuscript. LPI performed the phasiRNA analyses, participated in data interpretation and contributed to the drafting of the manuscript. FS and PP produced the sRNA libraries data. JLR, YFL and RS produced the degradome data and JLR contributed to the drafting of the manuscript. GH conceived and designed the whole project, contributed to the drafting of the manuscript and gave final approval of the version to be published. All authors read and approved the final manuscript.

\section{Acknowledgements}

JLR: Dirección General de Asuntos del Personal Académico (DGAPA), Universidad Nacional Autónoma de México (UNAM), (PAPIIT: IN205112) and Consejo Nacional de Ciencia y Tecnología (CONACyT) (no. 151571). GH: DGAPA-UNAM (PAPIIT: IN202213). DF: Postdoctoral fellowship from DGAPA-UNAM. LPI is a student in the Doctorado en Ciencias Biomédicas-UNAM and a recipient of a studentship from CONACyT (No.340334). We are grateful to Georgina Navarrete-Estrada from the Instituto de Biotecnología, UNAM for technical assistance in preparation of sRNA libraries, to Zamri Ramírez Carbajal for technical assistance in preparation of nodulation experiments and to Dr. Michael Dunn for critically reviewing the manuscript.

\section{Author details}

'Centro de Ciencias Genómicas, Universidad Nacional Autónoma de México (UNAM), Av. Universidad 1001, Cuernavaca 62210, Morelos, Mexico. 2Departamento de Biología Molecular de Plantas, Instituto de Biotecnología (UNAM), Av. Universidad 2001, Cuernavaca 62210, Morelos, Mexico. ${ }^{3}$ Department of Biochemistry and Molecular Biology, Oklahoma State University, Stillwater, OK 74078, USA.

Received: 7 January 2015 Accepted: 18 May 2015

Published online: 02 June 2015

\section{References}

1. Broughton WJ, Hernández G, Blair M, Beebe S, Gepts P, Vanderleyden J. Beans (Phaseolus spp.) - model food legumes. Plant Soil. 2003;252:55-128.

2. Schmutz J, McClean PE, Mamidi S, Wu GA, Cannon SB, Grimwood J, et al. A reference genome for common bean and genome-wide analysis of dual domestications. Nat Genet. 2014;46:707-13.

3. Kalavacharla V, Liu Z, Meyers BC, Thimmapuram J, Melmaiee K. Identification and analysis of common bean (Phaseolus vulgaris L.) transcriptomes by massively parallel pyrosequencing. BMC Plant Biol. 2011;11:135.

4. Liu Z, Crampton M, Todd A, Kalavacharla V. Identification of expressed resistance gene-like sequences by data mining in 454-derived transcriptomic sequences of common bean (Phaseolus vulgaris L.). BMC Plant Biol. 2012;12:42.

5. Hernández G, Valdés-López O, Ramírez M, Goffard N, Weiller G, Aparicio-Fabre R, et al. Global changes in the transcript and metabolic profiles during symbiotic nitrogen fixation in phosphorus-stressed common bean plants. Plant Physiol. 2009;151:1221-38.

6. Hiz MC, Canher B, Niron H, Turet M. Transcriptome analysis of salt tolerant common bean (Phaseolus vulgaris L.) under saline conditions. PLoS One. 2014:9:e92598.

7. Hernández G, Ramírez M, Valdés-López O, Tesfaye M, Graham MA, Czechowski T, et al. Phosphorus stress in common bean: root transcript and metabolic responses. Plant Physiol. 2007;144:752-67.

8. Valdés-López O, Yang SS, Aparicio-Fabre R, Graham PH, Reyes JL, Vance CP, et al. MicroRNA expression profile in common bean (Phaseolus vulgaris) under nutrient deficiency stresses and manganese toxicity. New Phytol. 2010;187:805-18.

9. Ramírez M, Flores-Pacheco G, Reyes JL, Luzlvarez A, Drevon JJ, Girard L, et al. Two Common Bean Genotypes with Contrasting Response to Phosphorus Deficiency Show Variations in the microRNA 399-Mediated PvPHO2 Regulation within the PvPHR1 Signaling Pathway. Int J Mol Sci. 2013;14:8328-44.

10. Naya L, Paul S, Valdés-López O, Mendoza-Soto AB, Nova-Franco B, Sosa-Valencia $\mathrm{G}$, et al. Regulation of copper homeostasis and biotic interactions by microRNA 398b in common bean. PLoS One. 2014:9:e84416.

11. Contreras-Cubas C, Rabanal FA, Arenas-Huertero C, Ortiz MA, Covarrubias AA, Reyes $J$. The Phaseolus vulgaris miR159a precursor encodes a second differentially expressed microRNA. Plant Mol Biol. 2012;80:103-15.

12. Jones-Rhoades MW, Bartel DP, Bartel B. MicroRNAs and their regulatory roles in plants. Annu Rev Plant Biol. 2006;57:19-53.

13. Li S-C, Chan W-C, Hu L-Y, Lai C-H, Hsu C-N, Lin W. Identification of homologous microRNAs in 56 animal genomes. Genomics. 2010;96:1-9.

14. Lin W-C, Li S-C, Lin W-C, Shin J-W, Hu S-N, Yu X-M, et al. Identification of microRNA in the protist Trichomonas vaginalis. Genomics. 2009:93:487-93.

15. Zhang $Y-Q$, Chen D-L, Tian H-F, Zhang B-H, Wen J-F. Genome-wide computational identification of microRNAs and their targets in the deep-branching eukaryote Giardia lamblia. Comput Biol Chem. 2009;33:391-6.

16. Voinnet O. Origin, biogenesis, and activity of plant microRNAs. Cell. 2009:136:669-87

17. Bartel DP. MicroRNAs: Genomics, biogenesis, mechanism, and function. Cell. 2004;116:281-97

18. Combier J-P, Frugier F, de Billy FF, Boualem A, El-Yahyaoui F, Moreau S, et al. MtHAP2-1 is a key transcriptional regulator of symbiotic nodule development regulated by microRNA169 in Medicago truncatula. Genes Dev. 2006;20:3084-8.

19. Chiou TJ, Aung K, Lin SI, Wu CC, Chiang SF, Su CL. Regulation of phosphate homeostasis by microRNA in Arabidopsis. Plant Cell. 2006;18:412-21.

20. Meng Y, Ma X, Chen D, Wu P, Chen M. MicroRNA-mediated signaling involved in plant root development. Biochem Biophys Res Commun. 2010;393:345-9. 
21. Navarro L, Dunoyer P, Jay F, Arnold B, Dharmasiri N, Estelle M, et al. A plant miRNA contributes to antibacterial resistance by repressing auxin signaling. Science (80). 2006;312:436-9.

22. Staiger D, Korneli C, Lummer M, Navarro L. Emerging role for RNA-based regulation in plant immunity. New Phytol. 2013;197:394-404.

23. Eckardt NA. The plant cell reviews aspects of microRNA and PhasiRNA regulatory function. Plant Cell. 2013;25:2382.

24. Allen E, Xie ZX, Gustafson AM, Carrington JC. microRNA-directed phasing during trans-acting siRNA biogenesis in plants. Cell. 2005;121:207-21.

25. Vazquez F, Vaucheret H, Rajagopalan R, Lepers C, Gasciolli V, Mallory AC, et al. Endogenous trans-acting siRNAs regulate the accumulation of Arabidopsis mRNAs. Mol Cell. 2004;16:69-79.

26. Song X, Li P, Zhai J, Zhou M, Ma L, Liu B, et al. Roles of DCL4 and DCL3b in rice phased small RNA biogenesis. Plant J. 2012;69:462-74.

27. Fei $Q$, Xia R, Meyers BC. Phased, secondary, small interfering RNAs in posttranscriptional regulatory networks. Plant Cell. 2013;25:2400-15.

28. Kozomara A, Griffiths-Jones S. miRBase: annotating high confidence microRNAs using deep sequencing data. Nucleic Acids Res. 2014:42(Database issue):D68-73.

29. Peláez $P$, Trejo MS, Iñiguez LP, Estrada-Navarrete G, Covarrubias AA, Reyes $J$, et al. Identification and characterization of microRNAs in Phaseolus vulgaris by high-throughput sequencing. BMC Genomics. 2012;13:83.

30. Han J, Xie H, Kong ML, Sun QP, Li RZ, Pan JB. Computational identification of miRNAs and their targets in Phaseolus vulgaris. Genet Mol Res. 2014:13:310-22.

31. Arenas-Huertero C, Pérez B, Rabanal F, Blanco-Melo D, De la Rosa C, Estrada-Navarrete $\mathrm{G}$, et al. Conserved and novel miRNAs in the legume Phaseolus vulgaris in response to stress. Plant Mol Biol. 2009;70:385-401.

32. Ormeño-Orrillo E, Menna P, Almeida LGP, Ollero FJ, Nicolás MF, Pains

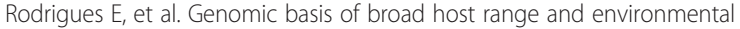
adaptability of Rhizobium tropici CIAT 899 and Rhizobium sp. PRF 81 which are used in inoculants for common bean (Phaseolus vulgaris L.). BMC Genomics. 2012;13:735

33. Yang $X$, Li L. miRDeep-P: a computational tool for analyzing the microRNA transcriptome in plants. Bioinformatics. 2011;27:2614-5.

34. Morin RD, O'Connor MD, Griffith M, Kuchenbauer F, Delaney A, Prabhu A-L, et al. Application of massively parallel sequencing to microRNA profiling and discovery in human embryonic stem cells. Genome Res. 2008;18:610-21.

35. Jones-Rhoades MW. Conservation and divergence in plant microRNAs. Plant Mol Biol. 2012;80:3-16

36. Li H, Deng Y, Wu T, Subramanian S, Yu O. Misexpression of miR482, miR1512, and miR1515 increases soybean nodulation. Plant Physiol. 2010;153:1759-70.

37. Bustos-Sanmamed P, Bazin J, Hartmann C, Crespi M, Lelandais-Briere C, Lelandais-Brière C. Small RNA pathways and diversity in model legumes: lessons from genomics. Front Plant Sci. 2013;4:236.

38. Zhu Q-H, Spriggs A, Matthew L, Fan L, Kennedy G, Gubler F, et al. A diverse set of microRNAs and microRNA-like small RNAs in developing rice grains. Genome Res. 2008;18:1456-65.

39. Lertpanyasampatha M, Gao L, Kongsawadworakul P, Viboonjun U, Chrestin $\mathrm{H}$, Liu R, et al. Genome-wide analysis of microRNAs in rubber tree (Hevea brasiliensis L.) using high-throughput sequencing. Planta. 2012;236:437-45.

40. Xia R, Meyers BC, Liu ZZ, Beers EP, Ye S, Liu ZZ. MicroRNA superfamilies descended from miR390 and their roles in secondary small interfering RNA Biogenesis in Eudicots. Plant Cell. 2013;25:1555-72.

41. Fahlgren N, Howell MD, Kasschau KD, Chapman EJ, Sullivan CM, Cumbie JS, et al. High-throughput sequencing of Arabidopsis microRNAs: evidence for frequent birth and death of MIRNA genes. PLoS One. 2007;2:e219.

42. Cuperus JT, Fahlgren N, Carrington JC. Evolution and functional diversification of MIRNA genes. Plant Cell. 2011;23:431-42.

43. Turner M, Yu O, Subramanian S. Genome organization and characteristics of soybean microRNAs. BMC Genomics. 2012;13:169

44. Formey D, Sallet E, Lelandais-Briere C, Ben CC, Bustos-Sanmamed P, Niebel $A$, et al. The small RNA diversity from Medicago truncatula roots under biotic interactions evidences the environmental plasticity of the miRNAome. Genome Biol. 2014;15:457.

45. O'Rourke JA, Iniguez LP, Fu F, Bucciarelli B, Miller SS, Jackson SA, et al. An RNA-Seq based gene expression atlas of the common bean. BMC Genomics. 2014;15:866

46. Addo-Quaye C, Snyder JA, Park YB, Li Y-F, Sunkar R, Axtell MJ. Sliced microRNA targets and precise loop-first processing of MIR319 hairpins revealed by analysis of the Physcomitrella patens degradome. RNA. 2009;15:2112-21.
47. Meng Y, Gou L, Chen D, Wu P, Chen M. High-throughput degradome sequencing can be used to gain insights into microRNA precursor metabolism. J Exp Bot. 2010;61:3833-7.

48. Meyers BC, Axtell MJ, Bartel B, Bartel DP, Baulcombe D, Bowman JL, et al Criteria for annotation of plant MicroRNAs. Plant Cell. 2008;20:3186-90.

49. Axtell MJ. Evolution of microRNAs and their targets: are all microRNAs biologically relevant? Biochim Biophys Acta. 2008;1779:725-34.

50. Zhuo Y, Gao G, Shi JA, Zhou X, Wang X. miRNAs: biogenesis, origin and evolution, functions on virus-host interaction. Cell Physiol Biochem. 2013;32:499-510

51. Colaiacovo M, Lamontanara A, Bernardo L, Alberici R, Crosatti C, Giusti L, et al. On the complexity of miRNA-mediated regulation in plants: novel insights into the genomic organization of plant miRNAs. Biol Direct. 2012;7:15.

52. Axtell MJ, Westholm JO, Lai EC. Vive la différence: biogenesis and evolution of microRNAs in plants and animals. Genome Biol. 2011;12:221.

53. Baskerville S, Bartel DP. Microarray profiling of microRNAs reveals frequent coexpression with neighboring miRNAs and host genes. RNA. 2005:11:241-7.

54. Elrouby N, Bureau TE. Bs1, a new chimeric gene formed by retrotransposonmediated exon shuffling in maize. Plant Physiol. 2010;153:1413-24.

55. Ma Z, Coruh C, Axtell MJ. Arabidopsis lyrata small RNAs: transient MIRNA and small interfering RNA loci within the Arabidopsis genus. Plant Cell. 2010;22:1090-103.

56. Li B, Duan H, Li J, Deng XW, Yin W, Xia X. Global identification of miRNAs and targets in Populus euphratica under salt stress. Plant Mol Biol. 2013;81:525-39.

57. Sun F, Guo G, Du J, Guo W, Peng H, Ni Z, et al. Whole-genome discovery of miRNAs and their targets in wheat (Triticum aestivum L.). BMC Plant Biol. 2014;14:142.

58. Xia R, Zhu H, An Y-Q, Beers EP, Liu Z. Apple miRNAs and tasiRNAs with novel regulatory networks. Genome Biol. 2012;13:R47.

59. Addo-Quaye C, Eshoo TW, Bartel DP, Axtell MJ. Endogenous siRNA and miRNA targets identified by sequencing of the Arabidopsis degradome. Curr Biol. 2008;18:758-62.

60. Dai X, Zhao PX. psRNATarget: a plant small RNA target analysis server Nucleic Acids Res. 2011;39(Web Server issue):W155-9.

61. Meyers BC, Green PJ. Plant MicroRNAs: Methods and Protocols, Humana pre. John M. Walker: Hatfield; 2010.

62. Rhoades MW, Reinhart BJ, Lim LP, Burge CB, Bartel B, Bartel DP. Prediction of plant microRNA targets. Cell. 2002;110:513-20.

63. Brandt R, Cabedo M, Xie Y, Wenkel S. Homeodomain leucine-zipper proteins and their role in synchronizing growth and development with the environment. J Integr Plant Biol. 2014;56:518-26.

64. Boualem A, Laporte P, Jovanovic M, Laffont C, Plet J, Combier J-P, et al. MicroRNA166 controls root and nodule development in Medicago truncatula. Plant J. 2008:54:876-87.

65. Langfelder P, Horvath S. WGCNA: an R package for weighted correlation network analysis. BMC Bioinformatics. 2008;9:559.

66. Valdés-López O, Arenas-Huertero C, Ramírez M, Girard L, Sánchez F, Vance $C P$, et al. Essential role of MYB transcription factor: PVPHR1 and microRNA: PvmiR399 in phosphorus-deficiency signalling in common bean roots. Plant Cell Environ. 2008:31:1834-43.

67. Liang $\mathrm{G}, \mathrm{He} H, \mathrm{Yu}$ D. Identification of nitrogen stanation-responsive microRNAs in Arabidopsis thaliana. PLoS One. 2012;7:e48951.

68. Olivera M, Tejera N, Iribarne C, Ocaña A, Lluch C. Effect of phosphorous on nodulation and nitrogen fixation by Phaseolus vulgaris. In: Velázquez $\mathrm{E}_{\text {, }}$ Rodríguez-Barrueco C, editors. First Int Meet Microb Phosphate Solubilization, vol. Volume 102. Dordrecht: Springer Netherlands; 2007. p. 157-60. Developments in Plant and Soil Sciences.

69. Awonaike KO, Lea PJ, Day JM, Roughley RJ, Miflin BJ. Effects of Combined Nitrogen on Nodulation and Growth of Phaseolus vulgaris. Exp Agric. 2008;16:303.

70. Bari R, Pant BD, Stitt M, Scheible W-R. PHO2, microRNA399, and PHR1 define a phosphate-signaling pathway in plants. Plant Physiol. 2006;141:988-99.

71. Zhai J, Jeong D-H, De Paoli E, Park S, Rosen BD, Li Y, et al. MicroRNAs as master regulators of the plant NB-LRR defense gene family via the production of phased, trans-acting siRNAs. Genes Dev. 2011;25:2540-53.

72. Brocker C, Vasiliou M, Carpenter S, Carpenter C, Zhang Y, Wang X, et al. Aldehyde dehydrogenase (ALDH) superfamily in plants: gene nomenclature and comparative genomics. Planta. 2013;237:189-210. 
73. Yoshida S, Kameoka H, Tempo M, Akiyama K, Umehara M, Yamaguchi S, et al. The D3 F-box protein is a key component in host strigolactone responses essential for arbuscular mycorrhizal symbiosis. New Phytol. 2012;196:1208-16.

74. Takahara M, Magori S, Soyano T, Okamoto S, Yoshida C, Yano K, et al. Too much love, a novel Kelch repeat-containing F-box protein, functions in the long-distance regulation of the legume-Rhizobium symbiosis. Plant Cell Physiol. 2013;54:433-47.

75. Arikit S, Xia R, Kakrana A, Huang K, Zhai J, Yan Z, et al. An atlas of soybean small RNAs identifies phased siRNAs from hundreds of coding genes. Plant Cell. 2014;26:4584-601.

76. Wang S, Sun X, Hoshino Y, Yu Y, Jia B, Sun Z, et al. MicroRNA319 positively regulates cold tolerance by targeting OsPCF6 and OsTCP21 in rice (Oryza sativa L.). PLoS One. 2014;9:e91357.

77. Zhou M, Luo H. Role of microRNA319 in creeping bentgrass salinity and drought stress response. Plant Signal Behav. 2014;9.

78. Schommer C, Bresso EG, Spinelli SV, Palatnik JF. Role of MicroRNA miR319 in Plant Development. In: Sunkar R, editor. MicroRNAs Plant Dev Stress Responses, vol. Volume 15. Berlin, Heidelberg: Springer Berlin Heidelberg; 2012. p. 29-47. Signaling and Communication in Plants.

79. De Luis A, Markmann K, Cognat V, Holt DB, Charpentier M, Parniske M, et al. Two microRNAs linked to nodule infection and nitrogen-fixing ability in the legume Lotus japonicus. Plant Physiol. 2012;160:2137-54

80. Ariel F, Brault-Hernandez M, Laffont C, Huault E, Brault M, Plet J, et al. Two direct targets of cytokinin signaling regulate symbiotic nodulation in Medicago truncatula. Plant Cell. 2012;24:3838-52.

81. Lauressergues D, Delaux P-MM, Formey D, Lelandais-Brière C, Fort S, Cottaz S, et al. The microRNA miR171h modulates arbuscular mycorrhizal colonization of Medicago truncatula by targeting NSP2. Plant J. 2012;72:512-22

82. Yan Z, Hossain MS, Wang J, Valdés-López O, Liang Y, Libault M, et al. miR172 regulates soybean nodulation. Mol Plant Microbe Interact. 2013;26:1371-7.

83. Nova-Franco B, Iñiguez LP, Valdés-López O, Alvarado-Affantranger X, Leija A, Fuentes SI, et al. The miR172C-AP2-1 Node as a Key Regulator of the Common Bean - Rhizobia Nitrogen Fixation Symbiosis. Plant Physiol. 2015; 114:255547

84. Wang Y, Wang L, Zou Y, Chen L, Cai Z, Zhang S, et al. Soybean miR172C Targets the Repressive AP2 Transcription Factor NNC1 to Activate ENOD40 Expression and Regulate Nodule Initiation. Plant Cell. 2014;26:4782-801.

85. Zhang S, Wang Y, Li K, Zou Y, Chen L, Li X. Identification of Cold-Responsive miRNAs and Their Target Genes in Nitrogen-Fixing Nodules of Soybean. Int J Mol Sci. 2014;15:13596-614.

86. Oldroyd GED, Downie JA. Calcium, kinases and nodulation signalling in legumes. Nat Rev Mol Cell Biol. 2004;5:566-76.

87. Montgomery TA, Howell MD, Cuperus JT, Li D, Hansen JE, Alexander AL, et al. Specificity of ARGONAUTE7-miR390 interaction and dual functionality in TAS3 trans-acting siRNA formation. Cell. 2008;133:128-41.

88. Howell MD, Fahlgren N, Chapman EJ, Cumbie JS, Sullivan CM, Givan SA, et al. Genome-wide analysis of the RNA-DEPENDENT RNA POLYMERASE6/ DICER-LIKE4 pathway in Arabidopsis reveals dependency on miRNA- and tasiRNA-directed targeting. Plant Cell. 2007;19:926-42.

89. Visser M, van der Walt AP, Maree HJ, Rees DJG, Burger JT. Extending the sRNAome of apple by next-generation sequencing. PLoS One. 2014;9:e95782

90. Liu Y, Wang Y, Zhu Q-H, Fan L. Identification of phasiRNAs in wild rice (Oryza rufipogon). Plant Signal Behav. 2013;8.

91. Manavella PA, Koenig D, Weigel D. Plant secondary siRNA production determined by microRNA-duplex structure. Proc Natl Acad Sci U S A. 2012:109:2461-6.

92. Liu $H$, Wang $X$, Zhang $H$, Yang $Y$, Ge $X$, Song F. A rice serine carboxypeptidase-like gene OsBISCPL1 is involved in regulation of defense responses against biotic and oxidative stress. Gene. 2008;420:57-65.

93. De Paoli E, Dorantes-Acosta A, Zhai J, Accerbi M, Jeong D-H, Park S, et al. Distinct extremely abundant siRNAs associated with cosuppression in petunia. RNA. 2009;15:1965-70.

94. Xie Q, Frugis G, Colgan D, Chua NH. Arabidopsis NAC1 transduces auxin signal downstream of TIR1 to promote lateral root development. Genes Dev. 2000;14:3024-36.

95. Broughton WJ, Dilworth MJ. Control of leghaemoglobin synthesis in snake beans. 1971
96. German MA, Luo S, Schroth G, Meyers BC, Green PJ. Construction of Parallel Analysis of RNA Ends (PARE) libraries for the study of cleaved miRNA targets and the RNA degradome. Nat Protoc. 2009;4:356-62.

97. Johnson M, Zaretskaya I, Raytselis Y, Merezhuk Y, McGinnis S, Madden TL. NCBI BLAST: a better web interface. Nucleic Acids Res. 2008;36:W5-9.

98. Li W, Godzik A. Cd-hit: a fast program for clustering and comparing large sets of protein or nucleotide sequences. Bioinformatics. 2006;22:1658-9.

99. Addo-Quaye C, Miller W, Axtell MJ. CleaveLand: a pipeline for using degradome data to find cleaved small RNA targets. Bioinformatics. 2009;25:130-1.

100. Ashburner M, Ball CA, Blake JA, Botstein D, Butler H, Cherry JM, et al. Gene ontology: tool for the unification of biology. The Gene Ontology Consortium. Nat Genet. 2000;25:25-9.

101. Zhi-Liang H, Bao J, Reecy J. CateGOrizer: A Web-Based Program to Batch Analyze Gene Ontology Classification Categories. Online J Bioinforma. 2008;9:108-12.

102. Edwards AWF. Cogwheels of the Mind: The Story of Venn Diagrams. JHU: JHU Press; 2004.

103. R Development Core Team. R: A Language and Environment for Statistical Computing. Vienna, Austria: R Foundation for Statistical Computing; 2009.

104. Shannon P, Markiel A, Ozier O, Baliga NS, Wang JT, Ramage D, et al. Cytoscape: a software environment for integrated models of biomolecular interaction networks. Genome Res. 2003;13:2498-504.

105. Langmead B, Trapnell C, Pop M, Salzberg SL. Ultrafast and memory-efficient alignment of short DNA sequences to the human genome. Genome Biol. 2009;10:R25.

106. Vandesompele J, De Preter K, Pattyn F, Poppe B, Van Roy N, De Paepe A, Speleman F: Accurate normalization of real-time quantitative RT-PCR data by geometric averaging of multiple internal control genes. Genome Biol 2002, 3.

\section{Submit your next manuscript to BioMed Central and take full advantage of:}

- Convenient online submission

- Thorough peer review

- No space constraints or color figure charges

- Immediate publication on acceptance

- Inclusion in PubMed, CAS, Scopus and Google Scholar

- Research which is freely available for redistribution 